J. Nonlinear Var. Anal. 5 (2021), No. 3, pp. 331-351

Available online at http://jnva.biemdas.com

https://doi.org/10.23952/jnva.5.2021.3.01

\title{
GENERIC CONVERGENCE OF METHODS FOR SOLVING STOCHASTIC FEASIBILITY PROBLEMS
}

\author{
KAY BARSHAD, SIMEON REICH*, ALEXANDER J. ZASLAVSKI \\ Department of Mathematics, The Technion - Israel Institute of Technology, 32000 Haifa, Israel
}

\begin{abstract}
We use an implementation of the generic approach to solve (generalized) stochastic feasibility problems. These are the problems of finding almost common fixed points of measurable (with respect to a probability measure) families of mappings. Such an implementation for a bounded set $K$ has already been presented by Gabour, Reich and Zaslavski in 2001. Our strong convergence results provide iterative methods (in the case where the set $K$ is not necessarily bounded) for finding an almost common fixed point of a generic measurable family of mappings. Some of our results involve the case where a subset of the almost common fixed point set is a nonexpansive retract of $K$. Our results are applicable to both the consistent case (that is, the case where the aforesaid almost common fixed points exist) and the inconsistent case (that is, the case where there are no common fixed points at all).

Keywords. Baire category; Banach space; Common fixed point problem; Residual set; Stochastic feasibility problem.
\end{abstract}

\section{INTRODUCTION AND BACKGROUND}

Suppose that $(X,\|\cdot\|)$ is a normed vector space with norm $\|\cdot\|, K \subset X$ is a nonempty, closed and convex subset of $X$, and $(\Omega, \mathscr{A}, \mu)$ is a probability measure space (more information on measure spaces and measurable mappings can be found, for example, in [1]). Denote by $\mathscr{N}$ the set of all bounded and nonexpansive mappings $A: K \rightarrow K$, that is, all bounded mappings $A: K \rightarrow K$ such that $\|A x-A y\| \leq\|x-y\|$ for each $x, y \in K$. For the set $\mathscr{N}$, define a metric $\rho_{\mathscr{N}}: \mathscr{N} \times \mathscr{N} \rightarrow \mathbb{R}$ by

$$
\rho_{\mathscr{N}}(A, B):=\sup \{\|A x-B x\|: x \in K\}, A, B \in \mathscr{N} .
$$

Clearly, the metric space $\left(\mathscr{N}, \rho_{\mathscr{N}}\right)$ is complete if $(X,\|\cdot\|)$ is a Banach space. Denote by $\mathscr{M}$ the set of all sequences $\left\{A_{n}\right\}_{n=1}^{\infty} \subset \mathscr{N}$. For the set $\mathscr{M}$, we consider two uniformities determined by the bases

$$
\begin{gathered}
E_{1}^{\prime}(N, \varepsilon)=\left\{\left(\left\{A_{n}\right\}_{n=1}^{\infty},\left\{B_{n}\right\}_{n=1}^{\infty}\right) \in \mathscr{M} \times \mathscr{M}: \rho_{\mathscr{N}}\left(\left\{A_{n}\right\}_{n=1}^{\infty},\left\{B_{n}\right\}_{n=1}^{\infty}\right)<\varepsilon, n=1,2, \ldots N\right\} \\
\quad \text { and } \\
E_{2}^{\prime}(\varepsilon)=\left\{\left(\left\{A_{n}\right\}_{n=1}^{\infty},\left\{B_{n}\right\}_{n=1}^{\infty}\right) \in \mathscr{M} \times \mathscr{M}: \rho_{\mathscr{N}}\left(\left\{A_{n}\right\}_{n=1}^{\infty},\left\{B_{n}\right\}_{n=1}^{\infty}\right)<\varepsilon, n=1,2, \ldots\right\},
\end{gathered}
$$

${ }^{*}$ Corresponding author.

E-mail addresses: kaybarshad@ campus.technion.ac.il (K. Barshad), sreich@technion.ac.il (S. Reich), ajzasl@ technion.ac.il (A.J. Zaslavski).

Received February 17, 2021; Accepted March 12, 2021.

(C)2021 Journal of Nonlinear and Variational Analysis 
where $\varepsilon>0$ and $N=1,2, \ldots$. These two uniformities induce two uniform topologies, respectively, $\tau_{1}^{\prime}$ and $\tau_{2}^{\prime}$ on $\mathscr{M}$. These topologies on $\mathscr{M}$ will be called the weak and strong topologies, respectively. Clearly, $\tau_{1}^{\prime}$ is indeed weaker than $\tau_{2}^{\prime}$, and the uniform space $\mathscr{M}$ with each of these topologies is metrizable (by metrics $\rho_{\tau_{1}^{\prime}}$ and $\rho_{\tau_{2}^{\prime}}$, respectively) and complete if $(X,\|\cdot\|)$ is a Banach space. More information on uniformities and uniform spaces can be found, for example, in [2].

Denote by $\mathscr{N}_{\Omega}$ the set of all bounded mappings $T: \Omega \rightarrow \mathscr{N}$ such that, for each $x \in K$, the mapping $T_{x}^{\prime}: \Omega \rightarrow K$, defined, for each $\omega \in \Omega$, by $T_{x}^{\prime}(\omega):=T(\omega)(x)$ for each $x \in K$, is measurable. It is not difficult to see that if $T \in \mathscr{N}_{\Omega}$, then $T_{x}^{\prime}$ is integrable on $\Omega$. For each $T \in \mathscr{N}_{\Omega}$, define an operator $\widetilde{T}: K \rightarrow K$ by $\widetilde{T} x=\int_{\Omega} T_{x}^{\prime}(\omega) d \mu(\omega)$. By the separation theorem, this is indeed a self-mapping of $K$. Note that this mapping is onto $K$. Clearly, for each $T \in \mathscr{N}_{\Omega}$, we have $\widetilde{T} \in \mathscr{N}$. Thus we consider the topology defined by the following pseudo-metric on $\mathscr{N}_{\Omega}$ :

$$
\rho_{\mathscr{N}_{\Omega}}(T, S):=\rho_{\mathscr{N}}(\widetilde{T}, \widetilde{S}), T, S \in \mathscr{N}_{\Omega} .
$$

It is not difficult to see that the pseudo-metric space $\left(\mathscr{N}_{\Omega}, \rho_{\mathscr{N}_{\Omega}}\right)$ is complete if $(X,\|\cdot\|)$ is a Banach space. The topology defined by the pseudo-metric $\rho_{\mathscr{N}_{\Omega}}$ on $\mathscr{N}_{\Omega}$ will be called the weak topology. For the set $\mathscr{N}_{\Omega}$, define a metric $d_{\mathscr{N}_{\Omega}}: \mathscr{N} \times \mathscr{N} \rightarrow \mathbb{R}$ by

$$
d_{\mathscr{N}_{\Omega}}(T, S):=\sup \left\{\rho_{\mathscr{N}}(T(\omega), S(\omega)): \omega \in \Omega\right\}, T, S \in \mathscr{N}_{\Omega} .
$$

The topology defined by the metric $d_{\mathscr{N}_{\Omega}}$ on $\mathscr{N}_{\Omega}$ will be called the strong topology. It is not difficult to see that this topology is indeed stronger than the topology defined on $\mathscr{N}_{\Omega}$ by the pseudo-metric $\rho_{\mathscr{N}_{\Omega}}$. Clearly, the metric space $\left(\mathscr{N}_{\Omega}, d_{\mathscr{N}_{\Omega}}\right)$ is complete if $(X,\|\cdot\|)$ is a Banach space.

Denote by $\mathscr{M}_{\Omega}$ the set of all sequences $\left\{T_{n}\right\}_{n=1}^{\infty} \subset \mathscr{N}_{\Omega}$. For the set $\mathscr{M}_{\Omega}$, we consider two uniformities determined by the bases

$$
\begin{gathered}
E_{1}(N, \varepsilon)=\left\{\left(\left\{T_{n}\right\}_{n=1}^{\infty},\left\{S_{n}\right\}_{n=1}^{\infty}\right) \in \mathscr{M}_{\Omega} \times \mathscr{M}_{\Omega}: \rho_{\mathscr{N}_{\Omega}}\left(T_{n}, S_{n}\right)<\varepsilon, n=1,2, \ldots N\right\} \\
\quad \text { and } \\
E_{2}(\varepsilon)=\left\{\left(\left\{T_{n}\right\}_{n=1}^{\infty},\left\{S_{n}\right\}_{n=1}^{\infty}\right) \in \mathscr{M}_{\Omega} \times \mathscr{M}_{\Omega}: \rho_{\mathscr{N}_{\Omega}}\left(T_{n}, S_{n}\right)<\varepsilon, n=1,2, \ldots\right\},
\end{gathered}
$$

where $\varepsilon>0$ and $N=1,2, \ldots$ These two uniformities induce two uniform topologies, $\tau_{1}$ and $\tau_{2}$, respectively, on $\mathscr{M}_{\Omega}$. These topologies on $\mathscr{M}_{\Omega}$ will be called the weak and strong topologies, respectively. Clearly, $\tau_{1}$ is indeed weaker than $\tau_{2}$, and the uniform space $\mathscr{M}_{\Omega}$ with each of these topologies is pseudo-metrizable (by pseudo-metrics $\rho_{\tau_{1}}$ and $\rho_{\tau_{2}}$, respectively) and complete if $(X,\|\cdot\|)$ is a Banach space.

We denote by $\mathscr{M}_{\Omega}^{b}$ the set of all sequences of mappings $\left\{T_{n}\right\}_{n=1}^{\infty} \subset \mathscr{N}_{\Omega}$ which are bounded in $\left(\mathscr{N}_{\Omega}, d_{\mathscr{N}_{\Omega}}\right)$ and by $\mathscr{M}_{\Omega}^{B}$ the set of all sequences of mappings $\left\{T_{n}\right\}_{n=1}^{\infty} \subset \mathscr{N}_{\Omega}$ which are bounded in $\left(\mathscr{N}_{\Omega}, \rho_{\mathscr{N}_{\Omega}}\right)$. It is not difficult to see that $\mathscr{M}_{\Omega}^{b} \subset \mathscr{M}_{\Omega}^{B}$. For the set $\mathscr{M}_{\Omega}^{b}$, define a metric $d_{\mathscr{M}_{\Omega}^{b}}: \mathscr{M}_{\Omega}^{b} \times \mathscr{M}_{\Omega}^{b} \rightarrow \mathbb{R}$ by

$$
d_{\mathscr{M}_{\Omega}^{b}}\left(\left\{T_{n}\right\}_{n=1}^{\infty},\left\{S_{n}\right\}_{n=1}^{\infty}\right):=\sup \left\{d_{\mathscr{N}_{\Omega}}\left(T_{n}, S_{n}\right): n=1,2 \ldots\right\},\left\{T_{n}\right\}_{n=1}^{\infty},\left\{S_{n}\right\}_{n=1}^{\infty} \in \mathscr{N}_{\Omega} .
$$

The topology defined by the metric $d_{\mathscr{M}_{\Omega}^{b}}$ on $\mathscr{M}_{\Omega}^{b}$ will be denoted by $\tau_{3}$. It is not difficult to see that this topology is stronger than the relative strong topology (and therefore stronger than the 
relative weak topology) on $\mathscr{M}_{\Omega}^{b}$. Clearly, the metric space $\left(\mathscr{M}_{\Omega}^{b}, d_{\mathscr{M}_{\Omega}^{b}}\right)$ is complete if $(X,\|\cdot\|)$ is a Banach space, and $\mathscr{M}_{\Omega}^{B}$ is a closed subset of $\mathscr{M}_{\Omega}$ with respect to the strong topology.

The following two propositions follow easily from the definitions.

Proposition 1.1. The mapping $T \rightarrow \widetilde{T}$ from $\mathscr{N}_{\Omega}$ with the weak topology onto $\left(\mathscr{N}, \rho_{\mathscr{N}}\right)$ is continuous.

Proposition 1.2. The mapping $\left\{T_{n}\right\}_{n=1}^{\infty} \rightarrow\left\{\widetilde{T}_{n}\right\}_{n=1}^{\infty}$ from $\mathscr{M}_{\Omega}$ with the strong (respectively, weak) topology onto $\mathscr{M}$ with the strong (respectively, weak) topology is continuous.

We denote by $\mathscr{N}_{\Omega}^{r e g}$ the set of all operators $T \in \mathscr{N}_{\Omega}$ for which there exists $x \in K$ such that $\widetilde{T} x=x$, and by $\mathscr{M}_{\Omega}^{\text {reg }}$ the set of all sequences $\left\{T_{n}\right\}_{n=1}^{\infty} \in \mathscr{M}_{\Omega}$ for which there exists $x \in K$ such that $\widetilde{T}_{n} x=x$ for all $n=1,2, \ldots$. We denote by $\overline{\mathscr{N}}_{\Omega}^{\text {reg }}$ the closure of $\mathscr{N}_{\Omega}^{\text {reg }}$ in $\mathscr{N}_{\Omega}$ with respect to the strong topology, by $\overline{\mathscr{M}}_{\Omega}^{\text {reg }} \cap \mathscr{M}_{\Omega}^{B}$ the closure of $\mathscr{M}_{\Omega}^{r e g} \cap \mathscr{M}_{\Omega}^{B}$ with respect to the strong topology and by $\overline{\mathscr{M}}_{\Omega}^{r e g} \cap \mathscr{M}_{\Omega}^{b}$ the closure of $\mathscr{M}_{\Omega}^{r e g} \cap \mathscr{M}_{\Omega}^{b}$ with respect to the $\tau_{3}$ topology.

Finally, we consider $F \subset K$, which is a nonempty, bounded, closed and convex subset of $K$. Denote by $\mathscr{N}_{\Omega}^{(F)}$ the set of all operators $T \in \mathscr{N}_{\Omega}$ such that, for almost all $\omega \in \Omega$, we have $T(\omega) x=x$ for each $x \in F$. It is not difficult to see that $\mathscr{N}_{\Omega}^{(F)}$ is a closed subset of $\mathscr{N}_{\Omega}$ with respect to the strong topology. Denote by $\mathscr{M}_{\Omega}^{(F)}$ the set of all sequences $\left\{T_{n}\right\}_{n=1}^{\infty} \in \mathscr{N}_{\Omega}^{(F)}$ and by $\overline{\mathscr{M}_{\Omega}^{(F)}}$ the closure of $\mathscr{M}_{\Omega}^{(F)}$ in $\mathscr{M}_{\Omega}$ with the weak topology. It is not difficult to see that $\mathscr{M}_{\Omega}^{(F)} \cap \mathscr{M}_{\Omega}^{b}$ is a closed subset of $\mathscr{M}_{\Omega}^{b}$ with respect to the $\tau_{3}$ topology. Denote by $\overline{\mathscr{M}_{\Omega}^{(F)} \cap \mathscr{M}_{\Omega}^{B}}$ the closure of $\mathscr{M}_{\Omega}^{(F)} \cap \mathscr{M}_{\Omega}^{B}$ with respect to the strong topology.

Recall that a mapping $P: K \rightarrow F$ is a nonexpansive retraction of $K$ onto $F$ if $P \in \mathscr{N}$ and $P x=x$ for all $x \in F$, and $F$ is a nonexpansive retract of $K$ if there exists a nonexpansive retraction $P$ of $K$ onto $F$. More information on nonexpansive retractions and nonexpansive retracts can be found, for example, in [3] and [4], and the references therein.

In all our results we also assume $(X,\|\cdot\|)$ is a Banach space.

\section{STATEMENTS OF THE MAIN RESUltS}

In this section, we state our main results. They are based on the generic approach (for more applications of this approach, see, for example, [5]). We establish them in Section 4 below. Some of our results include, inter alia, the extensions of all the results which were obtained in [6] to an unbounded set $K$.

Recall that, for each $S \in \mathscr{N}_{\Omega}$, a point $x \in K$ is an almost common fixed point of the family $\{S(\omega)\}_{\omega \in \Omega}$ if $S(\omega) x=x$ for almost all $\omega \in \Omega$. Similarly, for each $\left\{S_{n}\right\}_{n=1}^{\infty} \in \mathscr{M}_{\Omega}$, a point $x \in K$ is an almost common fixed point of the family $\left\{S_{n}(\omega)\right\}_{\omega \in \Omega, n=1,2 \ldots}$ if $S_{n}(\omega) x=x$ for all $n=1,2, \ldots$ and almost all $\omega \in \Omega$.

Theorem 2.1. There exist sets $\mathscr{F} \subset \mathscr{N}_{\Omega}$ and $\mathscr{F}^{\prime} \subset \mathscr{F} \cap \overline{\mathscr{N}_{\Omega}^{\text {reg }}}$, which are countable intersections of open (in the relative weak topology) and dense (respectively, in the strong topology and in the relative strong topology) subsets of, respectively, $\mathscr{N}_{\Omega}$ and $\overline{\mathscr{N}_{\Omega}^{\text {reg }}}$ so that, for each $S \in \mathscr{F}$, the following assertion holds: 
There exists $x_{S} \in K$, which is the unique fixed point of the operator $\widetilde{S}$, such that, for each $x \in K$, the sequence $\left\{\widetilde{S}^{n} x\right\}_{n=1}^{\infty}$ converges to $x_{S}$, uniformly on $K$, and the set of almost common fixed points of the family $\{S(\omega)\}_{\omega \in \Omega}$ is contained in $\left\{x_{S}\right\}$. Moreover, for each $\varepsilon>0$, there exist a positive integer $N$ and a neighborhood $U$ of $S$ in $\mathscr{N}_{\Omega}$ with the weak topology such that, for each integer $n \geq N$ and each $R \in U$,

$$
\left\|\widetilde{R}^{n} x-x_{S}\right\|<\varepsilon
$$

for each $x \in K$.

Theorem 2.2. There exist sets $\mathscr{F} \subset \overline{\mathscr{M}_{\Omega}^{\text {reg }} \cap \mathscr{M}_{\Omega}^{B}}$ and $\mathscr{F}^{\prime} \subset \mathscr{F} \cap \mathscr{M}_{\Omega}^{b}$, which are countable intersections of open (in the relative strong topology) and dense (respectively, in the relative strong topology and in the relative $\tau_{3}$ topology) subsets of, respectively, $\overline{\mathscr{M}}_{\Omega}^{\text {reg }} \cap \mathscr{M}_{\Omega}^{B}$ and $\overline{\mathscr{M}_{\Omega}^{\text {reg }} \cap \mathscr{M}_{\Omega}^{b}}$, so that for each $\left\{S_{n}\right\}_{n=1}^{\infty} \in \mathscr{F}$, there exists $x_{S} \in K$, which is the unique common fixed point of the operators ${\widetilde{S_{n}}}_{n}, n=1,2, \ldots$, such that for each $x \in K$, the sequence $\left\{{\widetilde{S_{n}}}^{k} x\right\}_{k=1}^{\infty}$ converges to $x_{S}$, uniformly on $K$, for each $n=1,2, \ldots$, and the set of almost common fixed points of the family $\left\{S_{n}(\omega)\right\}_{\omega \in \Omega, n=1,2 \ldots}$ is contained in $\left\{x_{S}\right\}$. Moreover, the following assertion holds:

For each $\varepsilon>0$, there exist a positive integer $N$ and a neighborhood $U$ of $\left\{S_{n}\right\}_{n=1}^{\infty}$ in $\mathscr{M}_{\Omega}$ with the strong topology such that, for each $\left\{R_{n}\right\}_{n=1}^{\infty} \in U$, each integer $n \geq N$ and each mapping $r:\{1, \ldots, n\} \rightarrow\{1,2, \ldots\}$,

$$
\left\|\widetilde{R_{r(n)}} \ldots \widetilde{R_{r(1)}} x-x_{S}\right\|<\varepsilon
$$

for each $x \in K$.

Theorem 2.3. There exist sets $\mathscr{F} \subset \mathscr{M}_{\Omega}, \mathscr{F}^{\prime} \subset \mathscr{F} \cap \mathscr{M}_{\Omega}^{B}$ and $\mathscr{F}^{\prime \prime} \subset \mathscr{F}^{\prime} \cap \mathscr{M}_{\Omega}^{b}$, which are countable intersections of open (in the relative weak topology) and dense (respectively, in the relative weak topology, in the relative strong topology and in the $\tau_{3}$ topology) subsets of, respectively, $\mathscr{M}_{\Omega}, \mathscr{M}_{\Omega}^{B}$, and $\mathscr{M}_{\Omega}^{b}$, so that for each $\left\{S_{n}\right\}_{n=1}^{\infty} \in \mathscr{F}$, the following assertion holds:

For each $\varepsilon>0$, there exist a positive integer $N$ and a neighborhood $U$ of $\left\{S_{n}\right\}_{n=1}^{\infty}$ in $\mathscr{M}_{\Omega}$ with the weak topology such that, for each $\left\{R_{n}\right\}_{n=1}^{\infty} \in U$ and each integer $n \geq N$,

$$
\left\|\widetilde{R_{n}} \ldots \widetilde{R_{1}} x-\widetilde{R_{n}} \ldots \widetilde{R_{1}} y\right\|<\varepsilon
$$

for all $x, y \in K$.

Theorem 2.4. There exist sets $\mathscr{F} \subset \mathscr{M}_{\Omega}^{B}$ and $\mathscr{F}^{\prime} \subset \mathscr{F} \cap \mathscr{M}_{\Omega}^{b}$, which are countable intersections of open (in the relative strong topology) and dense (respectively, in the relative strong topology and in the $\tau_{3}$ topology) subsets of, respectively, $\mathscr{M}_{\Omega}^{B}$ and $\mathscr{M}_{\Omega}^{b}$, so that for each $\left\{S_{n}\right\}_{n=1}^{\infty} \in \mathscr{F}$, the following assertion holds:

For each $\varepsilon>0$, there exist a positive integer $N$ and a neighborhood $U$ of $\left\{S_{n}\right\}_{n=1}^{\infty}$ in $\mathscr{M}_{\Omega}$ with the strong topology such that, for each $\left\{R_{n}\right\}_{n=1}^{\infty} \in U$, each integer $n \geq N$ and each mapping $r:\{1, \ldots, n\} \rightarrow\{1,2, \ldots\}$

$$
\left\|\widetilde{R_{r(n)}} \ldots \widetilde{R_{r(1)}} x-\widetilde{R_{r(n)}} \ldots \widetilde{R_{r(1)}} y\right\|<\varepsilon
$$

for each $x, y \in K$. 
Theorem 2.5. Assume $F$ is a nonexpansive retract of $K$. Then there exists a set $\mathscr{F} \subset \mathscr{N}_{\Omega}^{(F)}$, which is countable intersections of open (in the weak topology) and dense (in the strong topology) subsets of $\mathscr{N}_{\Omega}^{(F)}$, so that for each $S \in \mathscr{F}$, the set of almost common fixed points of the family $\{S(\omega)\}_{\omega \in \Omega}$ coincides with $F$ and there exists a nonexpansive retraction $Q$ of $K$ onto $F$ such that the following assertions hold:

(1) The sequence of operators $\left\{\widetilde{S}^{n}\right\}_{n=1}^{\infty}$ converges to $Q$, uniformly on $K$.

(2) For each $\varepsilon>0$, there exist a positive integer $N$ and a neighborhood $U$ of $S$ in $\mathscr{N}_{\Omega}^{(F)}$ with the weak topology such that, for each $R \in U$ and each integer $n \geq N$,

$$
\left\|\widetilde{R}^{n} x-Q x\right\|<\varepsilon
$$

for each $x \in K$.

Theorem 2.6. Assume $F$ is a nonexpansive retract of $K$. Then there exist sets $\mathscr{F} \subset \overline{\mathscr{M}_{\Omega}^{(F)}}$, $\mathscr{F}^{\prime} \subset \mathscr{F} \cap \mathscr{M}_{\Omega}^{B}$ and $\mathscr{F}^{\prime \prime} \subset \mathscr{F}^{\prime} \cap \mathscr{M}_{\Omega}^{b}$, which are countable intersections of open (in the relative weak topology) and dense (respectively, in the relative weak topology, in the relative strong topology and in the relative $\tau_{3}$ topology) subsets of, respectively, $\overline{\mathscr{M}_{\Omega}^{(F)}}, \overline{\mathscr{M}_{\Omega}^{(F)} \cap \mathscr{M}_{\Omega}^{B}}$ and $\mathscr{M}_{\Omega}^{(F)} \cap \mathscr{M}_{\Omega}^{b}$, so that for each $\left\{S_{n}\right\}_{n=1}^{\infty} \in \mathscr{F}$, the set of almost common fixed points of the family $\left\{S_{n}(\omega)\right\}_{\omega \in \Omega, n=1,2 \ldots}$ is contained in $F$ (if $\left\{S_{n}\right\}_{n=1}^{\infty} \in \mathscr{F}^{\prime \prime}$, then this set coincides with $F$ ) and there exists a nonexpansive retraction $Q$ of $K$ onto $F$ such that the following assertions hold:

(1) The sequence of operators $\left\{\widetilde{S_{n}} \ldots \widetilde{S_{1}}\right\}_{n=1}^{\infty}$ converges to $Q$, uniformly on $K$.

(2) For each $\varepsilon>0$, there exist a positive integer $N$ and a neighborhood $U$ of $\left\{S_{n}\right\}_{n=1}^{\infty}$ in $\overline{\mathscr{M}_{\Omega}^{(F)}}$ with the relative weak topology such that, for each $\left\{R_{n}\right\}_{n=1}^{\infty} \in U$ and each integer $n \geq N$,

$$
\left\|\widetilde{R_{n}} \ldots \widetilde{R_{1}} x-Q x\right\|<\varepsilon
$$

for each $x \in K$.

Theorem 2.7. Assume $F$ is a nonexpansive retract of $K$. Then there exist sets $\mathscr{F} \subset \overline{\mathscr{M}_{\Omega}^{(F)} \cap \mathscr{M}_{\Omega}^{B}}$ and $\mathscr{F}^{\prime} \subset \mathscr{F} \cap \mathscr{M}_{\Omega}^{b}$, which are countable intersections of open (in the relative strong topology) and dense (respectively, in the relative strong topology and in the relative $\tau_{3}$ topology) subsets of, respectively, $\mathscr{M}_{\Omega}^{(F)} \cap \mathscr{M}_{\Omega}^{B}$ and $\mathscr{M}_{\Omega}^{(F)} \cap \mathscr{M}_{\Omega}^{b}$, so that for each $\left\{S_{n}\right\}_{n=1}^{\infty} \in \mathscr{F}$, the set of almost common fixed points of the family $\left\{S_{n}(\omega)\right\}_{\omega \in \Omega, n=1,2 \ldots}$ is contained in $F$ (if $\left\{S_{n}\right\}_{n=1}^{\infty} \in \mathscr{F}^{\prime}$, then this set coincides with $F$ ) and the following assertions holds:

For each mapping $r:\{1,2, \ldots\} \rightarrow\{1,2, \ldots\}$, there exists a nonexpansive retraction $Q_{r}$ of $K$ onto $F$ such that:

(1) The sequence of operators $\left\{\widetilde{S_{r(n)}} \ldots \widetilde{S_{r(1)}}\right\}_{n=1}^{\infty}$ converges to $Q_{r}$, uniformly on $K$.

(2) For each $\varepsilon>0$, there exist a positive integer $N$ and a neighborhood $U$ of $\left\{S_{n}\right\}_{n=1}^{\infty}$ in $\overline{\mathscr{M}_{\Omega}^{(F)}}$ with the relative strong topology such that, for each $\left\{R_{n}\right\}_{n=1}^{\infty} \in U$ and each integer $n \geq N$,

$$
\left\|\widetilde{R_{r(n)}} \ldots \widetilde{R_{r(1)}} x-Q_{r} x\right\|<\varepsilon
$$


for each $x \in K$.

\section{Auxiliary Results}

In this section, we present several lemmata, which will be used in the proofs of our main results.

We begin by recalling that a family $E$ of operators $A: K \rightarrow K$ is said to be uniformly equicontinuous if for any $\varepsilon>0$, there exists $\delta>0$ such that $\|A x-A y\|<\varepsilon$ for each $A \in E$ and for each $x, y \in K$ satisfying $\|x-y\|<\delta$. The following lemma is a stronger version of Lemma 4.2 in [7].

Lemma 3.1. Assume $E$ is a nonempty uniformly equicontinuous set of operators $A: K \rightarrow K$, $N$ is a positive integer and $\varepsilon>0$ is a positive number. Then there exists a number $\delta>0$ such that for each sequence $\left\{A_{n}\right\}_{n=1}^{N} \subset E$ and each sequence $\left\{B_{n}\right\}_{n=1}^{N}$, where the (not necessarily continuous) operators $B_{n}: K \rightarrow K, n=1, \ldots, N$, satisfy, for each $n=1, \ldots N$,

$$
\left\|B_{n} z-A_{n} z\right\|<\delta
$$

for each $z \in K$, the following inequality holds

$$
\left\|B_{n} \ldots B_{1} x-A_{n} \ldots A_{1} x\right\|<\varepsilon
$$

for each $n=1, \ldots, N$ and each $x \in K$.

Proof. Define by recursion the following sequence of positive real numbers: $\varepsilon_{N}=\varepsilon$, and for each $n=1, \ldots, N-1$, choose $\varepsilon_{n} \leq \varepsilon_{n+1}$ such that, for each $x, y \in K$, with $\|x-y\|<\varepsilon_{n}$, we have $\|A x-A y\|<2^{-1} \varepsilon_{n+1}$ for each $A \in E$. Set $\delta:=2^{-1} \varepsilon_{1}$. Assume $\left\{A_{n}\right\}_{n=1}^{N} \subset E$ and that the (not necessarily continuous) operators $B_{n}: K \rightarrow K, n=1, \ldots, N$, satisfy (3.1). We now show by induction. For each $n=1, \ldots N$, we have

$$
\left\|B_{n} \ldots B_{1} x-A_{n} \ldots A_{1} x\right\|<\varepsilon_{n}, \quad \forall x \in K .
$$

For $n=1$, this follows from (3.1). For $n>1$, we suppose that

$$
\left\|B_{n-1} \ldots B_{1} x-A_{n-1} \ldots A_{1} x\right\|<\varepsilon_{n-1}
$$

for each $x \in K$. Then by the triangle inequality, (3.1), the definition of $\varepsilon_{n}$ and (3.2) we have

$$
\begin{gathered}
\quad\left\|B_{n} \ldots B_{1} x-A_{n} \ldots A_{1} x\right\| \leq\left\|B_{n} B_{n-1} \ldots B_{1} x-A_{n} B_{n-1} \ldots B_{1} x\right\| \\
+\left\|A_{n} B_{n-1} \ldots B_{1} x-A_{n} \ldots A_{1} x\right\|<\delta+2^{-1} \varepsilon_{n}=2^{-1}\left(\varepsilon_{1}+\varepsilon_{n}\right) \leq \varepsilon_{n}
\end{gathered}
$$

for each $x \in K$. Since, for each $n=1, \ldots, N, \varepsilon_{n} \leq \varepsilon$, the result follows.

Given $y^{*} \in K,\left\{A_{n}\right\}_{n=1}^{\infty} \in \mathscr{M}$ and $\gamma \in(0,1)$, we define a sequence of mappings $\left\{A_{n}^{\gamma}\right\}_{n=1}^{\infty}$, $A_{n}^{\gamma}: K \rightarrow K$ by

$$
A_{n}^{\gamma} x:=(1-\gamma) A_{n} x+\gamma y^{*}, n=1,2, \ldots
$$

for each $x \in K$. For each $n=1,2 \ldots$, we have

$$
\left\|A_{n}^{\gamma} x-A_{n}^{\gamma} y\right\| \leq(1-\gamma)\|x-y\|
$$

for each $x, y \in K$. Hence $\left\{A_{n}^{\gamma}\right\}_{n=1}^{\infty} \in \mathscr{M}$. It is not difficult to see that, for each sequence of operators $\left\{A_{n}\right\}_{n=1}^{\infty} \in \mathscr{M}$, the family of operators $\left\{\left\{A_{n}^{\gamma}\right\}_{n=1}^{\infty}: \gamma \in(0,1)\right\}$ is uniformly equicontinuous.

Denote by $\mathscr{M}^{B}$ the set of all sequences $\left\{A_{n}\right\}_{n=1}^{\infty} \in \mathscr{N}$, which are bounded in the space $\left(\mathscr{N}, \rho_{\mathscr{N}}\right)$. Clearly, for each $\left\{A_{n}\right\}_{n=1}^{\infty} \in \mathscr{M}^{B}$, we have $\left\{A_{n}^{\gamma}\right\}_{n=1}^{\infty} \in \mathscr{M}^{B}$. 


\subsection{Auxiliary results for Theorems 2.1 and 2.2.}

Lemma 3.2. Let $\varepsilon>0$ be an arbitrary positive number. Then for each $\left\{A_{n}\right\}_{n=1}^{\infty} \in \mathscr{M}^{B}$, each $\gamma \in(0,1)$ and each $y^{*} \in K$ satisfying $A_{n}^{\gamma_{\bar{x}}}=\bar{x}$ for some $\bar{x} \in K$ and all $n=1,2, \ldots$ (where $\left\{A_{n}^{\gamma}\right\}_{n=1}^{\infty}$ is defined by (3.3)), there exist a positive integer $N$ and a neighborhood $U$ of $\left\{A_{n}^{\gamma}\right\}_{n=1}^{\infty}$ in the space $\mathscr{M}$ with the strong topology such that the following assertion holds:

For each $\left\{B_{n}\right\}_{n=1}^{\infty} \in U$ and each mapping $r:\{1, \ldots, N\} \rightarrow\{1,2, \ldots\}$, we have

$$
\left\|B_{r(N)} \ldots B_{r(1)} x-\bar{x}\right\|<\varepsilon
$$

for all $x \in K$.

Proof. Let $\left\{A_{n}\right\}_{n=1}^{\infty} \in \mathscr{M}^{B}, \gamma \in(0,1)$ and $y^{*} \in K$ satisfy $A_{n}^{\gamma} \bar{x}=\bar{x}$ for some $\bar{x} \in K$ and all $n=$ $1,2, \ldots$ (where $\left\{A_{n}^{\gamma}\right\}_{n=1}^{\infty}$ is defined by (3.3)). Clearly, there exists $R_{0}>0$ such that $\left\|A_{n}^{\gamma} x\right\|<R_{0}$ for each $x \in K$ and all $n=1,2 \ldots$ Choose a positive integer $N>1$ such that

$$
(1-\gamma)^{N-1}\left(\|\bar{x}\|+R_{0}\right)<2^{-1} \varepsilon
$$

By Lemma 3.1, there exists a neighborhood $U$ of $\left\{A_{n}^{\gamma}\right\}_{n=1}^{\infty}$ in the space $\mathscr{M}$ with the strong topology such that, for each $\left\{B_{n}\right\}_{n=1}^{\infty} \in U$ and each mapping $r:\{1, \ldots, N\} \rightarrow\{1,2, \ldots\}$,

$$
\left\|B_{r(N)} \ldots B_{r(1)} x-A_{r(N)}^{\gamma} \ldots A_{r(1)}^{\gamma} x\right\|<2^{-1} \varepsilon
$$

for all $x \in K$. Let $\left\{B_{n}\right\}_{n=1}^{\infty} \in U$ and let $r:\{1, \ldots, N\} \rightarrow\{1,2, \ldots\}$ be an arbitrary mapping. Using (3.4) and induction, we get

$$
\left\|A_{r(N)}^{\gamma} \ldots A_{r(1)}^{\gamma} x-\bar{x}\right\|<2^{-1} \varepsilon
$$

for each $x \in K$. Inequalities (3.5) and (3.6) imply that

$$
\begin{gathered}
\left\|B_{r(N)} \ldots B_{1} x-\bar{x}\right\| \leq\left\|B_{r(N)} \ldots B_{r(1)} x-A_{r(N)}^{\gamma} \ldots A_{r(1)}^{\gamma} x\right\| \\
+\left\|A_{r(N)}^{\gamma} \ldots A_{r(1)}^{\gamma} x-\bar{x}\right\|<\varepsilon
\end{gathered}
$$

for each $x \in K$. Lemma 3.2 is proved.

For each $A \in \mathscr{N}, \gamma \in(0,1)$ and $y^{*} \in K$, define the mapping $A_{\gamma}: K \rightarrow K$ by

$$
A_{\gamma} x:=(1-\gamma) A x+\gamma y^{*}
$$

for each $x \in K$. Clearly,

$$
\left\|A_{\gamma} x-A_{\gamma} y\right\| \leq(1-\gamma)\|x-y\|
$$

for each $x, y \in K$ and $A_{\gamma} \in \mathscr{N}$. By Banach's fixed point theorem, there exists a unique $z\left(A, \gamma, y^{*}\right) \in$ $K$ such that for each $x \in K, A_{\gamma}^{n} x \rightarrow z\left(A, \gamma, y^{*}\right)$ as $n \rightarrow \infty$ and

$$
A_{\gamma z}\left(A, \gamma, y^{*}\right)=z\left(A, \gamma, y^{*}\right) \text {. }
$$

Using Lemma 3.2, we obtain the following result. 
Lemma 3.3. Let $\varepsilon>0$ be an arbitrary positive number. Then for each $A \in \mathscr{N}$, each $\gamma \in(0,1)$ and each $y^{*} \in K$, there exist a positive integer $N$ and a neighborhood $U$ of $A_{\gamma}$ (defined by (3.7)) in the space $\left(\mathscr{N}, \rho_{\mathscr{N}}\right)$ so that the following assertion holds:

For each $B \in U$ and each integer $n \geq N$, we have

$$
\left\|B^{n} x-z\left(A, \gamma, y^{*}\right)\right\|<\varepsilon
$$

for each $x \in K$.

Given $\left\{T_{n}\right\}_{n=1}^{\infty} \in \mathscr{M}_{\Omega}, \gamma \in(0,1)$ and $y^{*} \in K$, we define a sequence of mappings $\left\{T_{n}^{\gamma}\right\}_{n=1}^{\infty}$, $T_{n}^{\gamma}: \Omega \rightarrow \mathscr{N}$, by

$$
T_{n}^{\gamma}(\omega) x:=(1-\gamma) T_{n}(\omega) x+\gamma y^{*}, n=1,2, \ldots
$$

for each $\omega \in \Omega$ and each $x \in K$. Clearly, $\left\{T_{n}^{\gamma}\right\}_{n=1}^{\infty} \in \mathscr{M}_{\Omega}$. We have, for each $n=1,2, \ldots$,

$$
\begin{gathered}
\widetilde{T_{n}^{\gamma}} x=\int_{\Omega}\left((1-\gamma) T_{n}(\omega) x+\gamma y^{*}\right) d \mu(\omega)=\gamma y^{*}+(1-\gamma) \int_{\Omega} T_{n}(\omega) x d \mu(\omega) \\
=(1-\gamma) \widetilde{T}_{n} x+\gamma y^{*}
\end{gathered}
$$

for each $x \in K$. Therefore

$$
\widetilde{T_{n}^{\gamma}}=\widetilde{T}_{n}^{\gamma}=\widetilde{T}_{n \gamma}
$$

for each $n=1,2, \ldots$. We also have, for each $N=1,2, \ldots$,

$$
\rho_{\mathscr{N}_{\Omega}}\left(T_{n}^{\gamma}, T_{n}\right) \leq \gamma\left(\max \left\{\sup _{x \in K}\left\|\widetilde{T}_{k} x\right\|\right\}_{k=1}^{N}+\left\|y^{*}\right\|\right)
$$

for each $n=1,2 \ldots N$. If, in addition, $\left\{T_{n}\right\}_{n=1}^{\infty} \in \mathscr{M}_{\Omega}^{B}$, then by (3.10), $\left\{T_{n}^{\gamma}\right\}_{n=1}^{\infty} \in \mathscr{M}_{\Omega}^{B}$ and for each $n=1,2 \ldots$, we have

$$
\rho_{\mathscr{N}_{\Omega}}\left(T_{n}^{\gamma}, T_{n}\right) \leq \gamma\left(\sup _{x \in K, k=1,2, \ldots}\left\|\widetilde{T}_{k} x\right\|+\left\|y^{*}\right\|\right) .
$$

In addition, if $\left\{T_{n}\right\}_{n=1}^{\infty} \in \mathscr{M}_{\Omega}^{b}$, then by (3.9), $\left\{T_{n}^{\gamma}\right\}_{n=1}^{\infty} \in \mathscr{M}_{\Omega}^{b}$ and for each $n=1,2 \ldots$, we have

$$
d_{\mathscr{M}_{\Omega}}\left(\left\{T_{n}^{\gamma}\right\}_{n=1}^{\infty},\left\{T_{n}\right\}_{n=1}^{\infty}\right) \leq \gamma\left(\sup _{x \in K, \omega \in \Omega, k=1,2, \ldots}\left\|T_{k}(\omega) x\right\|+\left\|y^{*}\right\|\right) .
$$

In particular, for each $T \in \mathscr{N}_{\Omega}, \gamma \in(0,1)$ and $y^{*} \in K$, the mapping $T_{\gamma}: \Omega \rightarrow K$, defined by

$$
T_{\gamma}(\omega) x:=(1-\gamma) T(\omega) x+\gamma y^{*}
$$

for each $\omega \in \Omega$ and each $x \in K$, satisfies $T_{\gamma} \in \mathscr{N}_{\Omega}$, as well as

$$
\widetilde{T}_{\gamma}=\widetilde{T}_{\gamma}
$$

and

$$
d_{\mathscr{N}_{\Omega}}\left(T_{\gamma}, T\right) \leq \gamma\left(\sup _{x \in K, \omega \in \Omega}\|T(\omega) x\|+\left\|y^{*}\right\|\right) .
$$

Comparing (3.15) to Lemma 3.3 and Proposition 1.1, we immediately obtain the following lemma. 
Lemma 3.4. Let $\varepsilon>0$ be an arbitrary positive number. Then for each $T \in \mathscr{N}_{\Omega}$, each $\gamma \in(0,1)$ and each $y^{*} \in K$, there exist a positive integer $N$ and a neighborhood $U$ of $T_{\gamma}$ (defined by (3.9)) in the space $\mathscr{N}_{\Omega}$ with the weak topology so that the following assertion holds:

For each $S \in U$ and each integer $n \geq N$, we have

$$
\left\|\widetilde{S}^{n} x-z\left(\widetilde{T}, \gamma, y^{*}\right)\right\|<\varepsilon
$$

for each $x \in K$.

Recall that $\mathscr{M}_{\Omega}^{r e g}$ is the set of all sequences $\left\{T_{n}\right\}_{n=1}^{\infty} \in \mathscr{M}_{\Omega}$ for which there exists a point $x \in K$ such that $\widetilde{T}_{n} x=x$ for all $n=1,2, \ldots$, and $\mathscr{N}_{\Omega}^{\text {reg }}$ is the set of all mappings $T \in \mathscr{N}_{\Omega}$ for which there exists $x \in K$ such that $\widetilde{T} x=x$.

Given $\left\{T_{n}\right\}_{n=1}^{\infty} \in \mathscr{M}_{\Omega}^{r e g}$ with $x_{\left\{T_{n}\right\}_{n=1}^{\infty}}$ such that $\widetilde{T}_{n} x_{\left\{T_{n}\right\}_{n=1}^{\infty}}=x_{\left\{T_{n}\right\}_{n=1}^{\infty}}$ for each $n=1,2, \ldots$ and $\gamma \in(0,1)$, we define a sequence of mappings $\left\{T_{n}^{\gamma}\right\}_{n=1}^{\infty}, T_{n}^{\gamma}: \Omega \rightarrow N$, by (3.9) with respect to $x_{\left\{T_{n}\right\}_{n=1}^{\infty}}$, that is, for each $\omega \in \Omega$,

$$
T_{n}^{\gamma}(\omega) x:=(1-\gamma) T_{n}(\omega) x+\gamma x_{\left\{T_{n}\right\}_{n=1}^{\infty}}, n=1,2, \ldots
$$

for each $x \in K$. Since $\widetilde{T}_{n} x_{\left\{T_{n}\right\}_{n=1}^{\infty}}=x_{\left\{T_{n}\right\}_{n=1}^{\infty}}$ for each $n=1,2, \ldots$, by using (3.10), we have

$$
\widetilde{T_{n}^{\gamma}} x_{\left\{T_{n}\right\}_{n=1}^{\infty}}=x_{\left\{T_{n}\right\}_{n=1}^{\infty}}
$$

for all $n=1,2, \ldots$, and hence $\left\{T_{n}^{\gamma}\right\}_{n=1}^{\infty} \in \mathscr{M}_{\Omega}^{\text {reg }}$.

In particular, given $T \in \mathscr{N}_{\Omega}^{r e g}$ with $x_{T}$ such that $\widetilde{T} x_{T}=x_{T}$ and $\gamma \in(0,1)$, we define a mapping $T_{\gamma}: \Omega \rightarrow N$ by (3.14) with respect to $x_{T}$, that is, for each $\omega \in \Omega$,

$$
T_{\gamma}(\omega) x:=(1-\gamma) T(\omega) x+\gamma x_{T}
$$

for each $x \in K$. Since $\widetilde{T} x_{T}=x_{T}$, by using (3.15), we have

$$
\widetilde{T}_{\gamma} x_{T}=x_{T}
$$

for all $n=1,2, \ldots$, and hence $T_{\gamma} \in \mathscr{N}_{\Omega}^{\text {reg }}$.

By Proposition 1.2, the mapping $\left\{T_{n}\right\}_{n=1}^{\infty} \rightarrow\left\{\widetilde{T}_{n}\right\}_{n=1}^{\infty}$ from $\mathscr{M}_{\Omega}^{B}$ with the relative strong (respectively, relative weak) topology onto $\mathscr{M}^{B}$ with the relative strong (respectively, relative weak) topology is continuous. Comparing this, (3.18) and (3.10) to Lemma 3.2, we immediately obtain the following lemma.

Lemma 3.5. Let $\varepsilon>0$ be an arbitrary positive number. Then for each $\left\{T_{n}\right\}_{n=1}^{\infty} \in \mathscr{M}_{\Omega}^{\text {reg }} \cap \mathscr{M}_{\Omega}^{B}$ and each $\gamma \in(0,1)$, there exist a positive integer $N$ and a neighborhood $U$ of $\left\{T_{n}^{\gamma}\right\}_{n=1}^{\infty}$ (defined by (3.17)) in the space $\mathscr{M}_{\Omega}$ with the strong topology such that the following assertion holds:

For each $\left\{S_{n}\right\}_{n=1}^{\infty} \in U$ and each mapping $r:\{1, \ldots, N\} \rightarrow\{1,2, \ldots\}$, we have

$$
\left\|\widetilde{S_{r(N)}} \ldots \widetilde{S_{r(1)}} x-x_{\left\{T_{n}\right\}_{n=1}^{\infty}}\right\|<\varepsilon
$$

for each $x \in K$. 


\subsection{Auxiliary results for Theorems 2.3 and 2.4 .}

Lemma 3.6. Let $\varepsilon>0$ be an arbitrary positive number. Then, for each $\left\{A_{n}\right\}_{n=1}^{\infty} \in \mathscr{M}$, each $\gamma \in(0,1)$ and each $y^{*} \in K$, there exist a positive integer $N$ and a neighborhood $U$ of $\left\{A_{n}^{\gamma}\right\}_{n=1}^{\infty}$ (defined by (3.3)) in the space $\mathscr{M}$ with the weak topology satisfying the following assertion:

For each $\left\{B_{n}\right\}_{n=1}^{\infty} \in U$ and each integer $n \geq N$, we have

$$
\left\|B_{n} \ldots B_{1} x-B_{n} \ldots B_{n} y\right\|<\varepsilon
$$

for each $x, y \in K$.

Proof. Assume $\left\{A_{n}\right\}_{n=1}^{\infty} \in \mathscr{M}, \gamma \in(0,1)$ and $y^{*} \in K$. Clearly, there exists $R_{0}>0$ such that $\left\|A_{1}^{\gamma} x\right\|<R_{0}$ for each $x \in K$. Choose a positive integer $N>1$ such that $(1-\gamma)^{N-1} R_{0}<6^{-1} \varepsilon$. By Lemma 3.1, there exists a neighborhood $U$ of $\left\{A_{n}^{\gamma}\right\}_{n=1}^{\infty}$ in the space $\mathscr{M}$ with the weak topology such that, for each $\left\{B_{n}\right\}_{n=1}^{\infty} \in U$,

$$
\left\|B_{N} \ldots B_{1} x-A_{N}^{\gamma} \ldots A_{1}^{\gamma} x\right\|<3^{-1} \varepsilon
$$

for each $x \in K$. Using (3.4) and induction, we obtain

$$
\left\|A_{N}^{\gamma} \ldots A_{1}^{\gamma} x-A_{N}^{\gamma} \ldots A_{1}^{\gamma} y\right\|<3^{-1} \varepsilon
$$

for each $x, y \in K$. Let $\left\{B_{n}\right\}_{n=1}^{\infty} \in U$. Since each one of the operators $B_{n}$ is nonexpansive, inequalities (3.19) and (3.20) imply that, for each integer $n \geq N$,

$$
\begin{gathered}
\left\|B_{n} \ldots B_{1} x-B_{n} \ldots B_{1} y\right\| \leq\left\|B_{N} \ldots B_{1} x-B_{N} \ldots B_{1} y\right\| \leq \\
\left\|B_{N} \ldots B_{1} x-A_{N}^{\gamma} \ldots A_{1}^{\gamma} x\right\|+\left\|A_{N}^{\gamma} \ldots A_{1}^{\gamma} x-A_{N}^{\gamma} \ldots A_{1}^{\gamma} y\right\| \\
+\left\|A_{N}^{\gamma} \ldots A_{1}^{\gamma} y-B_{N} \ldots B_{1} y\right\|<\varepsilon
\end{gathered}
$$

for each $x, y \in K$. Lemma 3.6 is proved.

Lemma 3.7. Let $\varepsilon>0$ be an arbitrary positive number. Then for each $\left\{A_{n}\right\}_{n=1}^{\infty} \in \mathscr{M}^{B}$, each $\gamma \in(0,1)$ and each $y^{*} \in K$, there exist a positive integer $N$ and a neighborhood $U$ of $\left\{A_{n}^{\gamma}\right\}_{n=1}^{\infty}$ (defined by (3.3)) in the space $\mathscr{M}$ with the strong topology satisfying the following assertion:

For each $\left\{B_{n}\right\}_{n=1}^{\infty} \in U$, each integer $n \geq N$ and each mapping $r:\{1, \ldots, n\} \rightarrow\{1,2, \ldots\}$, we have

$$
\left\|B_{r(n)} \ldots B_{r(1)} x-B_{r(n)} \ldots B_{r(1)} y\right\|<\varepsilon
$$

for each $x, y \in K$.

Proof. Let $\left\{A_{n}\right\}_{n=1}^{\infty} \in \mathscr{M}, \gamma \in(0,1), y^{*} \in K$. Clearly, there exists $R_{0}>0$ such that $\left\|A_{n}^{\gamma} x\right\|<R_{0}$ for each $x \in K$ and all $n=1,2 \ldots$ Choose a positive integer $N>1$ such that $(1-\gamma)^{N-1} R_{0}<$ $6^{-1} \varepsilon$. By Lemma 3.1, there exists a neighborhood $U$ of $\left\{A_{n}^{\gamma}\right\}_{n=1}^{\infty}$ in the space $\mathscr{M}$ with the strong topology such that for each $\left\{B_{n}\right\}_{n=1}^{\infty} \in U$ and each mapping $r:\{1, \ldots, N\} \rightarrow\{1,2, \ldots\}$, we have

$$
\left\|B_{r(N)} \ldots B_{r(1)} x-A_{r(N)}^{\gamma} \ldots A_{r(1)}^{\gamma} x\right\|<3^{-1} \varepsilon
$$

for each $x \in K$. Using (3.4) and induction, we get

$$
\left\|A_{r(N)}^{\gamma} \ldots A_{r(1)}^{\gamma} x-A_{r(N)}^{\gamma} \ldots A_{r(1)}^{\gamma} y\right\|<3^{-1} \varepsilon
$$


for each $x, y \in K$. Let $\left\{B_{n}\right\}_{n=1}^{\infty} \in U$ and let $r:\{1, \ldots, n\} \rightarrow\{1,2, \ldots\}$ be an arbitrary mapping. Since each one of the operators $B_{n}$ is nonexpansive, inequalities (3.21) and (3.22) imply that, for each integer $n \geq N$,

$$
\begin{gathered}
\left\|B_{r(n)} \ldots B_{1} x-B_{r(n)} \ldots B_{r(1)} y\right\| \leq\left\|B_{r(N)} \ldots B_{r(1)} x-B_{r(N)} \ldots B_{r(1)} y\right\| \leq \\
\left\|B_{r(N)} \ldots B_{r(1)} x-A_{r(N)}^{\gamma} \ldots A_{r(1)}^{\gamma} x\right\|+\left\|A_{r(N)}^{\gamma} \ldots A_{r(1)}^{\gamma} x-A_{r(N)}^{\gamma} \ldots A_{r(1)}^{\gamma} y\right\| \\
+\left\|A_{r(N)}^{\gamma} \ldots A_{r(1)}^{\gamma} y-B_{r(N)} \ldots B_{r(1)} y\right\|<\varepsilon
\end{gathered}
$$

for all $x, y \in K$. This completes the proof of Lemma 3.7.

Comparing (3.10) to Lemma 3.6, Lemma 3.7 and Proposition 1.2, we immediately obtain the following two lemmata.

Lemma 3.8. Let $\varepsilon>0$ be an arbitrary positive number. Then for each $\left\{T_{n}\right\}_{n=1}^{\infty} \in \mathscr{M}_{\Omega}$, each $\gamma \in(0,1)$ and each $y^{*} \in K$, there exist a positive integer $N$ and a neighborhood $U$ of $\left\{T_{n}^{\gamma}\right\}_{n=1}^{\infty}$ (defined by (3.9)) in the space $\mathscr{M}_{\Omega}$ with the weak topology satisfying the following assertion:

For each $\{S\}_{n=1}^{\infty} \in U$ and each integer $n \geq N$, we have

$$
\left\|\widetilde{S_{n}} \ldots \widetilde{S_{1}} x-\widetilde{S_{n}} \ldots \widetilde{S_{1}} y\right\|<\varepsilon
$$

for each $x, y \in K$.

Lemma 3.9. Let $\varepsilon>0$ be an arbitrary positive number. Then for each $\left\{T_{n}\right\}_{n=1}^{\infty} \in \mathscr{M}_{\Omega}^{B}$, each $\gamma \in(0,1)$ and each $y^{*} \in K$, there exist a positive integer $N$ and a neighborhood $U$ of $\left\{T_{n}^{\gamma}\right\}_{n=1}^{\infty}$ (defined by (3.9)) in the space $\mathscr{M}_{\Omega}$ with the strong topology satisfying the following assertion:

For each $\{S\}_{n=1}^{\infty} \in U$, each integer $n \geq N$ and each mapping $r:\{1, \ldots, n\} \rightarrow\{1,2, \ldots\}$, we have

$$
\left\|\widetilde{S_{r(n)}} \ldots \widetilde{S_{r(1)}} x-\widetilde{S_{r(n)}} \ldots \widetilde{S_{r(1)}} y\right\|<\varepsilon
$$

for each $x, y \in K$.

3.3. Auxiliary results for Theorems 2.5, 2.6 and 2.7. Assume that $F$ is a nonexpansive retract of $K$. Then there exists $P: K \rightarrow F$ such that $P \in \mathscr{N}$ and $P x=x$ for all $x \in F$. For each $x \in K$, set $d(x, F)=\inf _{y \in F}\|x-y\|$. Denote by $\mathscr{N}^{(F)}$ the set of all $A \in \mathscr{N}$ such that $A x=x$ for all $x \in F$. Denote by $\mathscr{M}^{(F)}$ the set of all sequences $\left\{A_{n}\right\}_{n=1}^{\infty} \subset \mathscr{N}^{(F)}$. It is not difficult to see that $\mathscr{M}^{(F)}$ is a closed subset of $\mathscr{M}$ with the weak topology. Given $\left\{A_{n}\right\}_{n=1}^{\infty} \in \mathscr{M}$ and $\gamma \in(0,1)$, we define a sequence of mappings $\left\{A_{n}^{\gamma}\right\}_{n=1}^{\infty}, A_{n}^{\gamma}: K \rightarrow K$, by

$$
A_{n}^{\gamma} x:=(1-\gamma) A_{n} x+\gamma P x, n=1,2, \ldots
$$

for each $x \in K$. Clearly, $\left\{A_{n}^{\gamma}\right\}_{n=1}^{\infty} \in \mathscr{M}$, and if $\left\{A_{n}\right\}_{n=1}^{\infty} \in \mathscr{M}^{(F)}$, then $\left\{A_{n}^{\gamma}\right\}_{n=1}^{\infty} \in \mathscr{M}^{(F)}$, as well as if $\left\{A_{n}\right\}_{n=1}^{\infty} \in \mathscr{M}^{B}$, then $\left\{A_{n}^{\gamma}\right\}_{n=1}^{\infty} \in \mathscr{M}^{B}$.

In the next three lemmata, we assume $\left\{A_{n}^{\gamma}\right\}_{n=1}^{\infty}$ is defined by (3.23).

Lemma 3.10. Let $\left\{A_{n}\right\}_{n=1}^{\infty} \in \mathscr{M}^{(F)}$ and $\gamma \in(0,1)$. Then for each $x \in K$ and each $n=1,2, \ldots$, we have $d\left(A_{n}^{\gamma} x, F\right) \leq(1-\gamma) d(x, F)$. 
Proof. Let $x \in K$ and let $n \geq 1$ be an integer. Then for each $\varepsilon>0$, there exists $y \in F$ such that $\|x-y\|<d(x, F)+\varepsilon$. We have $(1-\gamma) y+\gamma P x \in F$ and by (3.23),

$$
\begin{gathered}
d\left(A_{n}^{\gamma} x, F\right) \leq\left\|A_{n}^{\gamma} x-(1-\gamma) y-\gamma P x\right\|=(1-\gamma)\left\|A_{n} x-y\right\| \\
\leq(1-\gamma)\|x-y\| \leq(1-\gamma)(d(x, F)+\varepsilon) .
\end{gathered}
$$

Since $\varepsilon$ is an arbitrary positive number, we conclude that $d\left(A_{n}^{\gamma} x, F\right) \leq(1-\gamma) d(x, F)$. This completes the proof of Lemma 3.10.

Lemma 3.11. Let $\varepsilon>0$ be an arbitrary positive number. Then for each $\left\{A_{n}\right\}_{n=1}^{\infty} \in \mathscr{M}^{(F)}$ and each $\gamma \in(0,1)$, there exist a positive integer $N$ and a neighborhood $U$ of $\left\{A_{n}^{\gamma}\right\}_{n=1}^{\infty}$ in the space $\mathscr{M}$ with the weak topology satisfying the following assertion:

There exists a nonexpansive retraction $Q$ of $K$ onto $F$ such that, for each $\left\{B_{n}\right\}_{n=1}^{\infty} \in U$,

$$
\left\|B_{N} \ldots B_{1} x-Q x\right\|<\varepsilon
$$

for each $x \in K$.

Proof. Fix $y^{*} \in F$. Let $\left\{A_{n}\right\}_{n=1}^{\infty} \in \mathscr{M}^{(F)}$ and $\gamma \in(0,1)$. Clearly, there exists a number $R_{0}>0$ such that $\left\|A_{1}^{\gamma} x\right\|<R_{0}$ for each $x \in K$. Choose a positive integer $N>1$ such that

$$
(1-\gamma)^{N-1}\left(\left\|y^{*}\right\|+R_{0}\right)<2^{-1} \varepsilon
$$

By Lemma 3.1, there exists a neighborhood $U$ of $\left\{A_{n}^{\gamma}\right\}_{n=1}^{\infty}$ in the space $\mathscr{M}$ with the weak topology such that, for each $\left\{B_{n}\right\}_{n=1}^{\infty} \in U$,

$$
\left\|B_{N} \ldots B_{1} x-A_{N}^{\gamma} \ldots A_{1}^{\gamma} x\right\|<2^{-1} \varepsilon
$$

for each $x \in K$. Using Lemma 3.10 and induction, we infer that

$$
d\left(A_{N}^{\gamma} \ldots A_{1}^{\gamma} x, F\right) \leq(1-\gamma)^{N-1}\left\|A_{1}^{\gamma} x-y^{*}\right\|<2^{-1} \varepsilon
$$

for each $x \in K$. Therefore there exists a mapping $Q: K \rightarrow F$ such that

$$
\left\|A_{N}^{\gamma} \ldots A_{1}^{\gamma} x-Q x\right\|<2^{-1} \varepsilon
$$

for each $x \in K$. Inequalities (3.24) and (3.25) imply that, for each $\left\{B_{n}\right\}_{n=1}^{\infty} \in U$,

$$
\left\|B_{N} \ldots B_{1} x-Q x\right\| \leq\left\|B_{N} \ldots B_{1} x-A_{N}^{\gamma} \ldots A_{1}^{\gamma} x\right\|+\left\|A_{N}^{\gamma} \ldots A_{1}^{\gamma} x-Q x\right\|<\varepsilon
$$

for each $x \in K$. This completes the proof of Lemma 3.11.

Lemma 3.12. Let $\varepsilon>0$ be an arbitrary positive number. Then for each $\left\{A_{n}\right\}_{n=1}^{\infty} \in \mathscr{M}^{(F)} \cap \mathscr{M}^{B}$ and each $\gamma \in(0,1)$, there exist a positive integer $N$ and a neighborhood $U$ of $\left\{A_{n}^{\gamma}\right\}_{n=1}^{\infty}$ in the space $\mathscr{M}$ with the strong topology satisfying the following assertion:

For each mapping $r:\{1, \ldots, N\} \rightarrow\{1,2, \ldots\}$, there exists a nonexpansive retraction $Q_{r}$ of $K$ onto $F$ such that, for each $\left\{B_{n}\right\}_{n=1}^{\infty} \in U$,

$$
\left\|B_{r(N)} \ldots B_{r(1)} x-Q_{r} x\right\|<\varepsilon
$$

for each $x \in K$.

Proof. Fix $y^{*} \in F$. Let $\left\{A_{n}\right\}_{n=1}^{\infty} \in \mathscr{M}^{(F)} \cap \mathscr{M}^{B}$ and $\gamma \in(0,1)$. Clearly, there exists $R_{0}>0$ such that $\left\|A_{n}^{\gamma} x\right\|<R_{0}$ for each $x \in K$ and all $n=1,2 \ldots$ Choose a positive integer $N>1$ such that

$$
(1-\gamma)^{N-1}\left(\left\|y^{*}\right\|+R_{0}\right)<2^{-1} \varepsilon
$$


By Lemma 3.1, there exists a neighborhood $U$ of $\left\{A_{n}^{\gamma}\right\}_{n=1}^{\infty}$ in the space $\mathscr{M}$ with the strong topology such that for each $\left\{B_{n}\right\}_{n=1}^{\infty} \in U$ and each mapping $r:\{1, \ldots, N\} \rightarrow\{1,2, \ldots\}$, we have

$$
\left\|B_{r(N)} \ldots B_{r(1)} x-A_{r(N)}^{\gamma} \ldots A_{r(1)}^{\gamma} x\right\|<2^{-1} \varepsilon
$$

for each $x \in K$. Let $r:\{1, \ldots, N\} \rightarrow\{1,2, \ldots\}$ be an arbitrary mapping. Using Lemma 3.10 and induction, we see that

$$
d\left(A_{r(N)}^{\gamma} \ldots A_{r(1)}^{\gamma} x, F\right) \leq(1-\gamma)^{N-1}\left\|A_{r(1)}^{\gamma} x-y^{*}\right\|<2^{-1} \varepsilon
$$

for each $x \in K$. Therefore there exist a mapping $Q_{r}: K \rightarrow F$ such that

$$
\left\|A_{r(N)}^{\gamma} \ldots A_{r(1)}^{\gamma} x-Q_{r} x\right\|<2^{-1} \varepsilon
$$

for each $x \in K$. Inequalities (3.26) and (3.27) imply that, for each $\left\{B_{n}\right\}_{n=1}^{\infty} \in U$,

$$
\begin{gathered}
\left\|B_{r(N)} \ldots B_{1} x-Q_{r} x\right\| \leq\left\|B_{r(N)} \ldots B_{r(1)} x-A_{r(N)}^{\gamma} \ldots A_{r(1)}^{\gamma} x\right\| \\
+\left\|A_{r(N)}^{\gamma} \ldots A_{r(1)}^{\gamma} x-Q_{r} x\right\|<\varepsilon
\end{gathered}
$$

for each $x \in K$. This completes the proof of Lemma 3.12.

Recall that we denote by $\mathscr{N}_{\Omega}^{(F)}$ the set of all operators $T \in \mathscr{N}_{\Omega}$ such that $(\forall x \in F) T(\omega) x=x$ for almost each $\omega \in \Omega$, and by $\mathscr{M}_{\Omega}^{(F)}$ the set of all sequences $\left\{T_{n}\right\}_{n=1}^{\infty} \subset$ $\mathscr{N}_{\Omega}^{(F)}$. Clearly, for each $T \in \mathscr{N}_{\Omega}^{(F)}$ and each $\left\{T_{n}\right\}_{n=1}^{\infty} \in \mathscr{M}_{\Omega}^{(F)}$, we have $\widetilde{T} \in N^{(F)}$ and $\left\{\widetilde{T}_{n}\right\}_{n=1}^{\infty} \in$ $\mathscr{M}^{(F)}$.

Given $\left\{T_{n}\right\}_{n=1}^{\infty} \in \mathscr{M}_{\Omega}, \gamma \in(0,1)$ and a nonexpansive retraction $P$ of $K$ onto $F$, we define a sequence of mappings $\left\{T_{n}^{\gamma}\right\}_{n=1}^{\infty}, T_{n}^{\gamma}: \Omega \rightarrow N$ by

$$
T_{n}^{\gamma}(\omega) x:=(1-\gamma) T_{n}(\omega) x+\gamma P x, n=1,2, \ldots
$$

for each $\omega \in \Omega$ and each $x \in K$. It is clear that $\left\{T_{n}^{\gamma}\right\}_{n=1}^{\infty} \in \mathscr{M}_{\Omega}^{(F)}$ if $\left\{T_{n}\right\}_{n=1}^{\infty} \in \mathscr{M}_{\Omega}^{(F)}$, and for each $n=1,2, \ldots$,

$$
\widetilde{T_{n}^{\gamma}}=\widetilde{T}_{n}^{\gamma}
$$

where the sequence $\left\{\widetilde{T}_{n}^{\gamma}\right\}_{n=1}^{\infty}$ is defined, for each $n=1,2, \ldots$, as in (3.23). We also have, for each $N=1,2, \ldots$,

$$
\rho_{\mathscr{N}_{\Omega}}\left(T_{n}^{\gamma}, T_{n}\right) \leq \gamma\left(\max \left\{\sup _{x \in K}\left\|\widetilde{T}_{k} x\right\|\right\}_{k=1}^{N}+\sup _{x \in K}\|P x\|\right)
$$

for each $n=1,2, \ldots N$. If, in addition, $\left\{T_{n}\right\}_{n=1}^{\infty} \in \mathscr{M}_{\Omega}^{B}$, then by (3.29), $\left\{T_{n}^{\gamma}\right\}_{n=1}^{\infty} \in \mathscr{M}_{\Omega}^{B}$ and for each $n=1,2 \ldots$, we have

$$
\rho_{\mathscr{N}_{\Omega}}\left(T_{n}^{\gamma}, T_{n}\right) \leq \gamma\left(\sup _{x \in K, k=1,2, \ldots}\left\|\widetilde{T}_{k} x\right\|+\sup _{x \in K}\|P x\|\right) .
$$


In addition, if $\left\{T_{n}\right\}_{n=1}^{\infty} \in \mathscr{M}_{\Omega}^{b}$, then by (3.28), $\left\{T_{n}^{\gamma}\right\}_{n=1}^{\infty} \in \mathscr{M}_{\Omega}^{b}$ and for each $n=1,2 \ldots$, we have

$$
d_{\mathscr{M}_{\Omega}}\left(\left\{T_{n}^{\gamma}\right\}_{n=1}^{\infty},\left\{T_{n}\right\}_{n=1}^{\infty}\right) \leq \gamma\left(\sup _{x \in K, \omega \in \Omega, k=1,2, \ldots}\left\|T_{k}(\omega) x\right\|+\sup _{x \in K}\|P x\|\right) .
$$

In particular, for each $T \in \mathscr{N}_{\Omega}$ and $\gamma \in(0,1)$, the mapping $T_{\gamma}: \Omega \rightarrow K$ defined by

$$
T_{\gamma}(\omega) x:=(1-\gamma) T(\omega) x+\gamma P x
$$

for each $\omega \in \Omega$ and each $x \in K$, satisfies $T_{\gamma} \in \mathscr{N}_{\Omega}^{(F)}$ if $T \in \mathscr{N}_{\Omega}^{(F)}$, and $\widetilde{T}_{\gamma}=\widetilde{T}_{\gamma}$, as well as

$$
d_{\mathscr{N}_{\Omega}}\left(T_{\gamma}, T\right) \leq \gamma\left(\sup _{x \in K, \omega \in \Omega}\|T(\omega) x\|+\sup _{x \in K}\|P x\|\right) .
$$

Comparing (3.29) to Lemma 3.11, Lemma 3.12 and Proposition 1.2, we immediately obtain the following two lemmata. In these lemmata, we assume that $\left\{T_{n}^{\gamma}\right\}_{n=1}^{\infty}$ is defined by (3.28).

Lemma 3.13. Let $\varepsilon>0$ be an arbitrary positive number. Then for each $\left\{T_{n}\right\}_{n=1}^{\infty} \in \mathscr{M}_{\Omega}^{(F)}$ and each $\gamma \in(0,1)$, there exist a positive integer $N$ and a neighborhood $U$ of $\left\{T_{n}^{\gamma}\right\}_{n=1}^{\infty}$ in the space $\mathscr{M}_{\Omega}$ with the weak topology satisfying the following assertion:

There exists a nonexpansive retraction $Q$ of $K$ onto $F$ such that, for each $\left\{S_{n}\right\}_{n=1}^{\infty} \in U$,

$$
\left\|\widetilde{S_{N}} \ldots \widetilde{S_{1}} x-Q x\right\|<\varepsilon
$$

for each $x \in K$.

Lemma 3.14. Let $\varepsilon>0$ be an arbitrary positive number. Then for each $\left\{T_{n}\right\}_{n=1}^{\infty} \in \mathscr{M}_{\Omega}^{(F)} \cap \mathscr{M}_{\Omega}^{B}$ and each $\gamma \in(0,1)$, there exist a positive integer $N$ and a neighborhood $U$ of $\left\{T_{n}^{\gamma}\right\}_{n=1}^{\infty}$ in the space $\mathscr{M}_{\Omega}$ with the strong topology satisfying the following assertion:

For each mapping $r:\{1, \ldots, N\} \rightarrow\{1,2, \ldots\}$, there exists a nonexpansive retraction $Q_{r}$ of $K$ onto $F$ such that, for each $\left\{S_{n}\right\}_{n=1}^{\infty} \in U$,

$$
\left\|\widetilde{S_{r(N)}} \ldots \widetilde{S_{r(1)}} x-Q_{r} x\right\|<\varepsilon
$$

for each $x \in K$.

\section{PROOF OF THE MAIN RESUlTS}

Proof of Theorem 2.1. Fix $y^{*} \in K$. For each $T \in \mathscr{N}_{\Omega}$ and $\gamma \in(0,1)$, define $y_{T}^{*}$ by

$$
y_{T}^{*}:=\left\{\begin{array}{ll}
x_{T} & \text { If } T \in \mathscr{N}_{\Omega}^{r e g} \\
y^{*} & \text { otherwise }
\end{array},\right.
$$

where $x_{T}$ satisfies $T x_{T}=x_{T}$. Define $T_{\gamma}: \Omega \rightarrow N$ by

$$
T_{\gamma}(\omega) x:=(1-\gamma) T(\omega) x+\gamma y_{T}^{*}
$$

for each $\omega \in \Omega$ and each $x \in K$. Set $\bar{x}(T, \gamma)=z\left(\widetilde{T}, \gamma, y_{T}^{*}\right)$, where $z\left(\widetilde{T}, \gamma, y_{T}^{*}\right)$ is the unique element of $K$ satisfying

$$
\widetilde{T}_{\gamma} z\left(\widetilde{T}, \gamma, y_{T}^{*}\right)=z\left(\widetilde{T}, \gamma, y_{T}^{*}\right)
$$


(see also (3.8)). By (3.16), the sets

$$
\begin{gathered}
A=\left\{T_{\gamma}: T \in \mathscr{N}_{\Omega}, \gamma \in(0,1)\right\} \\
\text { and } \\
A^{r e g}=\left\{T_{\gamma}: T \in \mathscr{N}_{\Omega}^{r e g}, \gamma \in(0,1)\right\}
\end{gathered}
$$

are dense, respectively, in $\mathscr{N}_{\Omega}$ with the strong topology and in $\overline{\mathscr{N}_{\Omega}^{\text {reg }}}$ with the relative strong topology.

Assume that $q$ is a positive integer. By Lemma 3.4, for each $(T, \gamma) \in \mathscr{N}_{\Omega} \times(0,1)$, there exist a positive integer $N_{q}(T, \gamma)$ and an open neighborhood $U_{q}(T, \gamma)$ of $T_{\gamma}$ in the space $\mathscr{N}_{\Omega}$ with the weak topology so that the following assertion holds:

For each $R \in U_{q}(T, \gamma)$ and each integer $n \geq N_{q}(T, \gamma)$, we have

$$
\left\|\widetilde{R}^{n} x-\bar{x}(T, \gamma)\right\|<\frac{1}{q}
$$

for all $x \in K$. For each positive integer $q$, set

$$
\begin{gathered}
\mathscr{D}_{q}=\cup_{(T, \gamma) \in \mathscr{N}_{\Omega} \times(0,1)} U_{q}(T, \gamma) \\
\text { and } \\
\mathscr{D}_{q}^{r e g}=\cup_{(T, \gamma) \in \overline{\mathscr{N}}_{\Omega}^{\text {reg }} \times(0,1)} U_{q}(T, \gamma) \cap \overline{\mathscr{N}_{\Omega}^{r e g}} .
\end{gathered}
$$

Clearly, the sets $\mathscr{D}_{q}$, and $\mathscr{D}_{q}^{\text {reg }}$ are open (in the relative weak topology) and dense (respectively, in the strong topology and in the relative strong topology) sets in, respectively, $\mathscr{N}_{\Omega}$ and $\overline{\mathscr{N}_{\Omega}^{r e g}}$ for each positive integer $q$, because the set $\mathscr{D}_{q}$ contains $A$ and the set $\mathscr{D}_{q}^{\text {reg }}$ contains $A^{\text {reg }}$. Define $\mathscr{F}=\cap_{q=1}^{\infty} \mathscr{D}_{q}$ and $\mathscr{F}^{\prime}=\cap_{q=1}^{\infty} \mathscr{D}_{q}^{r e g}$. Evidently, $\mathscr{F}$ and $\mathscr{F}^{\prime}$ are countable intersections of open (in the relative weak topology) and dense (respectively, in the strong topology and in the relative strong topology) sets in, respectively, $\mathscr{N}_{\Omega}$ and $\overline{\mathscr{N}_{\Omega}^{\text {reg }}}$.

Assume that $S \in \mathscr{F}$ and $\varepsilon>0$. There exist a positive integer $q_{0}$ such that $\frac{2}{q_{0}}<\varepsilon$ and a pair $(T, \gamma) \in \mathscr{N}_{\Omega} \times(0,1)$ such that $S \in U_{q_{0}}(T, \gamma)$. Let $R \in U_{q_{0}}(T, \gamma)$. By (4.1), for each positive integer $n \geq N_{q_{0}}(T, \gamma)$, we have

$$
\left\|\widetilde{R}^{n} x-\bar{x}(T, \gamma)\right\|<\frac{1}{q_{0}}<2^{-1} \varepsilon
$$

for each $x \in K$. Since $R \in U_{q_{0}}(T, \gamma)$ is arbitrary, we have, in particular, for each positive integer $n \geq N_{q_{0}}(T, \gamma)$

$$
\left\|\widetilde{S}^{n} x-\bar{x}(T, \gamma)\right\|<\frac{1}{q_{0}}<2^{-1} \varepsilon
$$

for all $x \in K$. Since $\varepsilon$ is an arbitrary positive number, (4.3), the triangle inequality and the completeness of the subspace $K$ of $X$ imply that the sequence of operators $\left\{\widetilde{S}^{n}\right\}_{n=1}^{\infty}$ converges uniformly to an operator $G: K \rightarrow K$. By switching to the limit in (4.3) and using the triangle inequality, a similar argument shows that $G$ must be constant, that is, there is $x_{S} \in K$ such that for each $x \in K, \widetilde{S}^{n} x \rightarrow x_{S}$, as $n \rightarrow \infty$, uniformly on $K$. By the continuity of the operator $\widetilde{S}$, we have

$$
\widetilde{S} x_{S}=\widetilde{S} \lim _{n \rightarrow \infty} \widetilde{S}^{n} x_{S}=\lim _{n \rightarrow \infty} \widetilde{S}^{n+1} x_{S}=x_{S}
$$


Therefore $x_{S}$ is the unique fixed point of the operator $\widetilde{S}$. Clearly, the set of almost common fixed points of the family $\left\{S_{\omega}\right\}_{\omega \in \Omega}$ is contained in $\left\{x_{S}\right\}$. By switching to the limit in (4.3), we see that

$$
\left\|x_{S}-\bar{x}(T, \gamma)\right\|<2^{-1} \varepsilon \text {. }
$$

Combining (4.4) with (4.2) and using the triangle inequality, we infer that for each integer $n \geq N_{q_{0}}(T, \gamma)$, we have $\left\|\widetilde{R}^{n} x-x_{S}\right\|<\varepsilon$ for all $x \in K$. This completes the proof of Theorem 2.1. Proof of Theorem 2.2. For each $\gamma \in(0,1)$ and $\left\{T_{n}\right\}_{n=1}^{\infty} \in \mathscr{M}_{\Omega}^{\text {reg }}$ with $x_{\left\{T_{n}\right\}_{n=1}^{\infty}}$ such that $T_{n} x_{\left\{T_{n}\right\}_{n=1}^{\infty}}=$

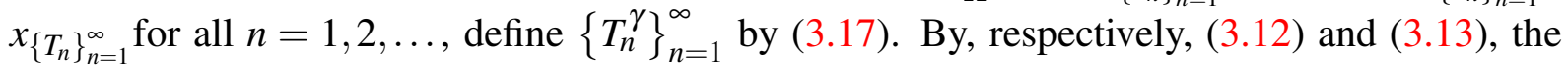
sets

$$
\begin{aligned}
& A^{B}=\left\{\left\{T_{n}^{\gamma}\right\}_{n=1}^{\infty}:\left\{T_{n}\right\}_{n=1}^{\infty} \in \mathscr{M}_{\Omega}^{r e g} \cap \mathscr{M}_{\Omega}^{B}, \gamma \in(0,1)\right\} \\
& \text { and } \\
& A^{b}=\left\{\left\{T_{n}^{\gamma}\right\}_{n=1}^{\infty}:\left\{T_{n}\right\}_{n=1}^{\infty} \in \mathscr{M}_{\Omega}^{r e g} \cap \mathscr{M}_{\Omega}^{b}, \gamma \in(0,1)\right\}
\end{aligned}
$$

are dense in, respectively, $\overline{\mathscr{M}_{\Omega}^{\text {reg }} \cap \mathscr{M}_{\Omega}^{B}}$ with the relative strong topology and $\overline{\mathscr{M}_{\Omega}^{\text {reg }} \cap \mathscr{M}_{\Omega}^{b}}$ with the relative $\tau_{3}$ topology.

Assume that $q$ is a positive integer. By Lemma 3.5, for each $\left(\left\{T_{n}\right\}_{n=1}^{\infty}, \gamma\right) \in\left(\mathscr{M}_{\Omega}^{r e g} \cap \mathscr{M}_{\Omega}^{B}\right) \times$ $(0,1)$, there exist a positive integer $N_{q}\left(\left\{T_{n}\right\}_{n=1}^{\infty}, \gamma\right)$ and an open neighborhood $U_{q}\left(\left\{T_{n}\right\}_{n=1}^{\infty}, \gamma\right)$ of $\left\{T_{n}^{\gamma}\right\}_{n=1}^{\infty}$ in the space $\mathscr{M}_{\Omega}$ with the strong topology, satisfying the following assertion:

For each $\left\{R_{n}\right\}_{n=1}^{\infty} \in U_{q}\left(\left\{T_{n}\right\}_{n=1}^{\infty}, \gamma\right)$ and each mapping $r:\left\{1, \ldots, N_{q}\left(\left\{T_{n}\right\}_{n=1}^{\infty}, \gamma\right)\right\} \rightarrow\{1,2, \ldots\}$, we have

$$
\left\|R_{r\left(N_{q}\left(\left\{T_{n}\right\}_{n=1}^{\infty}, \gamma\right)\right)} \ldots \widetilde{R_{r(1)}} x-x_{\left\{T_{n}\right\}_{n=1}^{\infty}}\right\|<\frac{1}{q}
$$

for each $x \in K$. For each positive integer $q$, set

$$
\begin{gathered}
\mathscr{D}_{q}^{B}=\cup_{\left(\left\{T_{n}\right\}_{n=1}^{\infty}, \gamma\right) \in\left(\mathscr{M}_{\Omega}^{r e g} \cap \mathscr{M}_{\Omega}^{B}\right) \times(0,1)} U_{q}\left(\left\{T_{n}\right\}_{n=1}^{\infty}, \gamma\right) \cap \overline{\mathscr{M}_{\Omega}^{r e g} \cap \mathscr{M}_{\Omega}^{B}} \\
\text { and } \\
\mathscr{D}_{q}^{b}=\cup_{\left(\left\{T_{n}\right\}_{n=1}^{\infty}, \gamma\right) \in\left(\mathscr{M}_{\Omega}^{r e g} \cap \mathscr{M}_{\Omega}^{b}\right) \times(0,1)} U_{q}\left(\left\{T_{n}\right\}_{n=1}^{\infty}, \gamma\right) \cap \overline{\mathscr{M}_{\Omega}^{r e g} \cap \mathscr{M}_{\Omega}^{b}} .
\end{gathered}
$$

Clearly, the sets $\mathscr{D}_{q}^{B}$ and $\mathscr{D}_{q}^{b}$ are open (in the relative strong topology) and dense (respectively, in the relative strong topology and in the relative $\tau_{3}$ topology) sets in, respectively, $\overline{\mathscr{M}_{\Omega}^{\text {reg }} \cap \mathscr{M}_{\Omega}^{B}}$ and $\overline{\mathscr{M}_{\Omega}^{r e g} \cap \mathscr{M}_{\Omega}^{b}}$ for each positive integer $q$, because these sets contain, respectively, $A^{B}$ and $A^{b}$. Define $\mathscr{F}=\cap_{q=1}^{\infty} \mathscr{D}_{q}^{B}$ and $\mathscr{F}^{\prime}=\cap_{q=1}^{\infty} \mathscr{D}_{q}^{b}$. Evidently, $\mathscr{F}$ and $\mathscr{F}^{\prime}$ are countable intersections of open (in the relative strong topology) and dense (respectively, in the relative strong topology and in the relative $\tau_{3}$ topology) sets in, respectively, $\overline{\mathscr{M}_{\Omega}^{\text {reg }} \cap \mathscr{M}_{\Omega}^{B}}$ and $\overline{\mathscr{M}_{\Omega}^{\text {reg }} \cap \mathscr{M}_{\Omega}^{b}}$.

Assume $\left\{S_{n}\right\}_{n=1}^{\infty} \in \mathscr{F}$ and $\varepsilon>0$. There exist a positive integer $q_{0}$ such that $\frac{4}{q_{0}}<\varepsilon$ and a pair $\left(\left\{T_{n}\right\}_{n=1}^{\infty}, \gamma\right) \in\left(\mathscr{M}_{\Omega}^{r e g} \cap \mathscr{M}_{\Omega}^{B}\right) \times(0,1)$ such that $\left\{S_{n}\right\}_{n=1}^{\infty} \in U_{q_{0}}\left(\left\{T_{n}\right\}_{n=1}^{\infty}, \gamma\right)$. Let $\left\{R_{n}\right\}_{n=1}^{\infty} \in$ $U_{q_{0}}\left(\left\{T_{n}\right\}_{n=1}^{\infty}, \gamma\right)$. By (4.5), for each mapping $r:\left\{1, \ldots, N_{q_{0}}\left(\left\{T_{n}\right\}_{n=1}^{\infty}, \gamma\right)\right\} \rightarrow\{1,2, \ldots\}$, we have

$$
\left\|R_{r\left(N_{q_{0}}\left(\left\{T_{n}\right\}_{n=1}^{\infty}, \gamma\right)\right)} \ldots \widetilde{R_{r(1)}} x-x_{\left\{T_{n}\right\}_{n=1}^{\infty}}\right\|<\frac{1}{q_{0}}<4^{-1} \varepsilon
$$


for each $x \in K$. In particular, for each $n=1,2, \ldots$, we have

$$
\left\|{\widetilde{R_{n}}}^{N_{q_{0}}\left(\left\{T_{n}\right\}_{n=1}^{\infty}, \gamma\right)} x-x_{\left\{T_{n}\right\}_{n=1}^{\infty}}\right\|<\frac{1}{q_{0}}<4^{-1} \varepsilon
$$

for all $x \in K$. Clearly, for each integer $k \geq N_{q_{0}}\left(\left\{T_{n}\right\}_{n=1}^{\infty}, \gamma\right)$ and each $n=1,2, \ldots$, we have

$$
\left\|{\widetilde{R_{n}}}^{k} x-x_{\left\{T_{m}\right\}_{m=1}^{\infty}}\right\|<\frac{1}{q_{0}}<4^{-1} \varepsilon
$$

for each $x \in K$. Since $\varepsilon$ is an arbitrary positive number, (4.7), the triangle inequality and the completeness of the subspace $K$ of $X$ imply that for each $n=1,2, \ldots$, the sequence of operators $\left\{{\widetilde{R_{n}}}^{k}\right\}_{k=1}^{\infty}$ converges uniformly to an operator $G_{n}: K \rightarrow K$. By switching to the limit in (4.7) and using the triangle inequality, a similar argument shows that $G_{n_{1}}=G_{n_{2}}$ for all positive integers $n_{1}$ and $n_{2}$, and the operator $G_{n}$ must be constant for each $n=1,2, \ldots$, that is, there is $x_{R} \in K$ such that for each $x \in K,{\widetilde{R_{n}}}^{k} x \rightarrow x_{R}$ as $k \rightarrow \infty$, uniformly on $K$, for each $n=1,2, \ldots$ By the continuity of the operator $\widetilde{R_{n}}$, for each $n=1,2, \ldots$,

$$
\widetilde{R_{n}} x_{R}=\widetilde{R_{n}} \lim _{k \rightarrow \infty}{\widetilde{R_{n}}}^{k} x=\lim _{k \rightarrow \infty}{\widetilde{R_{n}}}^{k+1} x_{R}=x_{R} .
$$

Therefore $x_{R}$ is the unique common fixed point of the operators $\widetilde{R_{n}}, n=1,2, \ldots$ Since $\left\{R_{n}\right\}_{n=1}^{\infty} \in$ $U_{q_{0}}\left(\left\{T_{n}\right\}_{n=1}^{\infty}, \gamma\right)$ is arbitrary, there is, in particular, $x_{S}$ which is the unique common fixed point of operators $\widetilde{S_{n}}, n=1,2, \ldots$, such that for each $x \in K,{\widetilde{S_{n}}}^{k} x \rightarrow x_{S}$ as $k \rightarrow \infty$, uniformly on $K$, for each $n=1,2, \ldots$. Clearly, the set of almost common fixed points of the family $\left\{S_{n}(\omega)\right\}_{\omega \in \Omega, n=1,2 \ldots}$ is contained in $\left\{x_{S}\right\}$. For each $n=1,2, \ldots$, each integer $k \geq N_{q_{0}}\left(\left\{T_{n}\right\}_{n=1}^{\infty}, \gamma\right)$ and each $n=1,2, \ldots$, we have from (4.7) that

$$
\left\|{\widetilde{S_{n}}}^{k} x-x_{\left\{T_{m}\right\}_{m=1}^{\infty}}\right\|<\frac{1}{q_{0}}<4^{-1} \varepsilon
$$

for each $x \in K$. By switching to the limit in (4.7), we also have

$$
\left\|x_{R}-x_{\left\{T_{n}\right\}_{n=1}^{\infty}}\right\|<4^{-1} \varepsilon .
$$

By switching to limit in (4.8), we have

$$
\left\|x_{S}-x_{\left\{T_{n}\right\}_{n=1}^{\infty}}\right\|<4^{-1} \varepsilon
$$

Combining (4.10) with (4.9) and using the triangle inequality, we obtain

$$
\left\|x_{R}-x_{S}\right\|<2^{-1} \varepsilon \text {. }
$$

By (4.6), (4.9), (4.11) and the triangle inequality, we infer that for each integer $n \geq N_{q_{0}}\left(\left\{T_{n}\right\}_{n=1}^{\infty}, \gamma\right)$ and each mapping $r:\{1, \ldots, n\} \rightarrow\{1,2, \ldots\}$, we have

$$
\begin{aligned}
& \left\|\widetilde{R_{r(n)}} \ldots \widetilde{R_{r(1)}} x-x_{S}\right\| \leq\left\|\widetilde{R_{r(n)}} \ldots \widetilde{R_{r(1)}} x-x_{R}\right\|+\left\|x_{R}-x_{S}\right\| \\
& \leq\left\|R_{r\left(N_{q_{0}}\left(\left\{T_{n}\right\}_{n=1}^{\infty}, \gamma\right)\right)} \cdots \widetilde{R_{r(1)}} x-x_{R}\right\|+\left\|x_{R}-x_{S}\right\| \\
& \leq\left\|R_{r\left(N_{q_{0}}\left(\left\{T_{n}\right\}_{n=1}^{\infty}, \gamma\right)\right)} \ldots \widetilde{R_{r(1)}} x-x_{\left\{T_{n}\right\}_{n=1}^{\infty}}\right\|+\left\|x_{\left\{T_{n}\right\}_{n=1}^{\infty}}-x_{R}\right\|+\left\|x_{R}-x_{S}\right\|<\varepsilon
\end{aligned}
$$

for each $x \in K$. This completes the proof of Theorem 2.2. 
Proof of Theorem 2.3. Fix $y^{*} \in K$. For each $\left\{T_{n}\right\}_{n=1}^{\infty} \in \mathscr{M}_{\Omega}$ and $\gamma \in(0,1)$, define $\left\{T_{n}^{\gamma}\right\}_{n=1}^{\infty}$ by (3.9). By, respectively, (3.11), (3.12) and (3.13), the sets

$$
\begin{aligned}
& A=\left\{\left\{T_{n}^{\gamma}\right\}_{n=1}^{\infty}:\left\{T_{n}\right\}_{n=1}^{\infty} \in \mathscr{M}_{\Omega}, \gamma \in(0,1)\right\}, \\
& A^{B}=\left\{\left\{T_{n}^{\gamma}\right\}_{n=1}^{\infty}:\left\{T_{n}\right\}_{n=1}^{\infty} \in \mathscr{M}_{\Omega}^{B}, \gamma \in(0,1)\right\} \\
& \text { and } \\
& A^{b}=\left\{\left\{T_{n}^{\gamma}\right\}_{n=1}^{\infty}:\left\{T_{n}\right\}_{n=1}^{\infty} \in \mathscr{M}_{\Omega}^{b}, \gamma \in(0,1)\right\}
\end{aligned}
$$

are dense in, respectively, $\mathscr{M}_{\Omega}$ with the relative weak topology, $\mathscr{M}_{\Omega}^{B}$ with the relative strong topology and $\mathscr{M}_{\Omega}^{b}$ with the $\tau_{3}$ topology. Assume that $q$ is a positive integer. By Lemma 3.8, for each $\left(\left\{T_{n}\right\}_{n=1}^{\infty}, \gamma\right) \in \mathscr{M}_{\Omega} \times(0,1)$, there exist a positive integer $N_{q}\left(\left\{T_{n}\right\}_{n=1}^{\infty}, \gamma\right)$ and an open neighborhood $U_{q}\left(\left\{T_{n}\right\}_{n=1}^{\infty}, \gamma\right)$ of $\left\{T_{n}^{\gamma}\right\}_{n=1}^{\infty}$ in the space $\mathscr{M}_{\Omega}$ with the weak topology, satisfying the following assertion:

For each $\left\{R_{n}\right\}_{n=1}^{\infty} \in U_{q}\left(\left\{T_{n}\right\}_{n=1}^{\infty}, \gamma\right)$ and each integer $n \geq N_{q}\left(\left\{T_{n}\right\}_{n=1}^{\infty}, \gamma\right)$, we have

$$
\left\|\widetilde{R_{n}} \ldots \widetilde{R_{1}} x-\widetilde{R_{n}} \ldots \widetilde{R_{1}} y\right\|<\frac{1}{q}
$$

for each $x, y \in K$. For each positive integer $q$, set

$$
\begin{gathered}
\mathscr{D}_{q}=\cup_{\left(\left\{T_{n}\right\}_{n=1}^{\infty}, \gamma\right) \in \mathscr{M}_{\Omega} \times(0,1)} U_{q}\left(\left\{T_{n}\right\}_{n=1}^{\infty}, \gamma\right), \\
\mathscr{D}_{q}^{B}=\cup_{\left(\left\{T_{n}\right\}_{n=1}^{\infty}, \gamma\right) \in \mathscr{M}_{\Omega}^{B} \times(0,1)} U_{q}\left(\left\{T_{n}\right\}_{n=1}^{\infty}, \gamma\right) \cap \mathscr{M}_{\Omega}^{B} \\
\text { and } \\
\mathscr{D}_{q}^{b}=\cup_{\left(\left\{T_{n}\right\}_{n=1}^{\infty}, \gamma\right) \in \mathscr{M}_{\Omega}^{b} \times(0,1)} U_{q}\left(\left\{T_{n}\right\}_{n=1}^{\infty}, \gamma\right) \cap \mathscr{M}_{\Omega}^{b} .
\end{gathered}
$$

Clearly, the sets $\mathscr{D}_{q}, \mathscr{D}_{q}^{B}$ and $\mathscr{D}_{q}^{b}$ are open (in the relative weak topology) and dense (respectively, in the weak topology, in the relative strong topology and in the relative $\tau_{3}$ topology) sets in, respectively, $\mathscr{M}_{\Omega}, \mathscr{M}_{\Omega}^{B}$ and $\mathscr{M}_{\Omega}^{b}$ for each positive integer $q$, because these sets contain, respectively, $A, A^{B}$ and $A^{b}$. Define $\mathscr{F}=\cap_{q=1}^{\infty} \mathscr{D}_{q}, \mathscr{F}^{\prime}=\cap_{q=1}^{\infty} \mathscr{D}_{q}^{B}$ and $\mathscr{F}^{\prime \prime}=\cap_{q=1}^{\infty} \mathscr{D}_{q}^{b}$. Evidently, $\mathscr{F}, \mathscr{F}^{\prime}$ and $\mathscr{F}^{\prime \prime}$ are countable intersections of open (in the relative weak topology) and dense (respectively, in the weak topology, in the relative strong topology and in the relative $\tau_{3}$ topology) sets in, respectively, $\mathscr{M}_{\Omega}, \mathscr{M}_{\Omega}^{B}$ and $\mathscr{M}_{\Omega}^{b}$. Assume $\left\{S_{n}\right\}_{n=1}^{\infty} \in \mathscr{F}$ and $\varepsilon>0$. There exists a positive integer $q_{0}$ such that $\frac{1}{q_{0}}<\varepsilon$ and a pair $\left(\left\{T_{n}\right\}_{n=1}^{\infty}, \gamma\right) \in \mathscr{M}_{\Omega} \times(0,1)$ such that $\left\{S_{n}\right\}_{n=1}^{\infty} \in U_{q_{0}}\left(\left\{T_{n}\right\}_{n=1}^{\infty}, \gamma\right)$. By (4.12), for each $\left\{R_{n}\right\}_{n=1}^{\infty} \in U_{q_{0}}\left(\left\{T_{n}\right\}_{n=1}^{\infty}, \gamma\right)$ and each integer $n \geq N_{q_{0}}\left(\left\{T_{n}\right\}_{n=1}^{\infty}, \gamma\right)$, we have

$$
\left\|\widetilde{R_{n}} \ldots \widetilde{R_{1}} x-\widetilde{R_{n}} \ldots \widetilde{R_{1}} y\right\|<\frac{1}{q_{0}}<\varepsilon
$$

for each $x, y \in K$. This completes the proof of Theorem 2.3.

The proof of Theorem 2.4 is analogous to that of Theorem 2.3. Instead of Lemma 3.8, we use Lemma 3.9.

Proof of Theorem 2.5. For each $T \in \mathscr{N}_{\Omega}^{(F)}$ and $\gamma \in(0,1)$, define $T_{\gamma}$ by (3.33). By (3.34), the set

$$
A=\left\{T_{\gamma}: T \in \mathscr{N}_{\Omega}^{(F)}, \gamma \in(0,1)\right\}
$$


is dense in $\mathscr{N}_{\Omega}^{(F)}$ with the relative strong topology. Assume that $q$ is a positive integer. By Lemma 3.13, for each $(T, \gamma) \in \mathscr{N}_{\Omega}^{(F)} \times(0,1)$, there exist a positive integer $N_{q}(T, \gamma)$ and an open neighborhood $U_{q}(T, \gamma)$ of $T_{\gamma}$ in the space $\mathscr{N}_{\Omega}$ with the weak topology satisfying the following assertion:

There exists a nonexpansive retraction $Q_{q}(T, \gamma)$ of $K$ onto $F$ such that for each $R \in U_{q}(T, \gamma)$, we have

$$
\left\|\widetilde{R}^{N_{q}(T, \gamma)} x-Q_{q}(T, \gamma) x\right\|<\frac{1}{q}
$$

for each $x \in K$. For each positive integer $q$, set

$$
\mathscr{D}_{q}=\cup_{(T, \gamma) \in \mathscr{N}_{\Omega}^{(F)} \times(0,1)} U_{q}(T, \gamma) \cap \mathscr{N}_{\Omega}^{(F)} .
$$

Clearly, the set $\mathscr{D}_{q}$ is open (in the weak topology) and dense (in the strong topology) set in $\mathscr{N}_{\Omega}^{(F)}$ for each positive integer $q$, because this set contains $A$. Define $\mathscr{F}=\cap_{q=1}^{\infty} \mathscr{D}_{q}$. Evidently, $\mathscr{F}$ is a countable intersection of open (in the weak topology) and dense (in the strong topology) sets in $\mathscr{N}_{\Omega}^{(F)}$. Assume that $S \in \mathscr{F}$ and $\varepsilon>0$. There exist a positive integer $q_{0}$ such that $\frac{2}{q_{0}}<\varepsilon$ and a pair $(T, \gamma) \in \mathscr{N}_{\Omega}^{(F)} \times(0,1)$ such that $S \in U_{q_{0}}(T, \gamma)$. Let $R \in U_{q_{0}}(T, \gamma) \cap \mathscr{N}_{\Omega}^{(F)}$. By (4.13), we have

$$
\left\|\widetilde{R}^{N_{q_{0}}(T, \gamma)} x-Q_{q_{0}}(T, \gamma) x\right\|<\frac{1}{q_{0}}<2^{-1} \varepsilon
$$

for each $x \in K$. Clearly, for each integer $n \geq N_{q_{0}}(T, \gamma)$, we have

$$
\left\|\widetilde{R}^{n} x-Q_{q_{0}}(T, \gamma) x\right\|<\frac{1}{q_{0}}<2^{-1} \varepsilon
$$

for each $x \in K$. In particular, for each integer $n \geq N_{q_{0}}(T, \gamma)$, we have

$$
\left\|\widetilde{S}^{n} x-Q_{q_{0}}(T, \gamma) x\right\|<\frac{1}{q_{0}}<2^{-1} \varepsilon
$$

for each $x \in K$. Since $\varepsilon$ is an arbitrary positive number, (4.15), the triangle inequality and the completeness of the subspace $K$ of $X$ imply that the sequence of operators $\left\{\widetilde{S}^{n}\right\}_{n=1}^{\infty}$ converges to an operator $Q: K \rightarrow K$, uniformly on $K$. By switching to the limit in (4.15), we have

$$
\left\|Q x-Q_{q_{0}}(T, \gamma) x\right\|<2^{-1} \varepsilon
$$

for each $x \in K$. Since $\varepsilon$ is an arbitrary positive number, using (4.16) and the closedness of $F$, we see that the image of $Q$ lies in $F$. By (4.14), (4.16) and the triangle inequality, we obtain that, for each integer $n \geq N_{q_{0}}(T, \gamma)$,

$$
\left\|\widetilde{R}^{n} x-Q x\right\| \leq\left\|\widetilde{R}^{n} x-Q_{q_{0}}(T, \gamma) x\right\|+\left\|Q_{q_{0}}(T, \gamma) x-Q x\right\|<\varepsilon
$$

for each $x \in K$. This completes the proof of Theorem 2.5. 
Proof of Theorem 2.6. For each $\left\{T_{n}\right\}_{n=1}^{\infty} \in \mathscr{M}_{\Omega}$ and $\gamma \in(0,1)$, define $\left\{T_{n}^{\gamma}\right\}_{n=1}^{\infty}$ by (3.28). By (3.30), (3.31) and (3.32), the sets

$$
\begin{gathered}
A=\left\{\left\{T_{n}^{\gamma}\right\}_{n=1}^{\infty}:\left\{T_{n}\right\}_{n=1}^{\infty} \in \mathscr{M}_{\Omega}^{(F)}, \gamma \in(0,1)\right\}, \\
A^{B}=\left\{\left\{T_{n}^{\gamma}\right\}_{n=1}^{\infty}:\left\{T_{n}\right\}_{n=1}^{\infty} \in \mathscr{M}_{\Omega}^{(F)} \cap \mathscr{M}_{\Omega}^{B}, \gamma \in(0,1)\right\} \\
\text { and } \\
A^{b}=\left\{\left\{T_{n}^{\gamma}\right\}_{n=1}^{\infty}:\left\{T_{n}\right\}_{n=1}^{\infty} \in \mathscr{M}_{\Omega}^{(F)} \cap \mathscr{M}_{\Omega}^{b}, \gamma \in(0,1)\right\}
\end{gathered}
$$

are dense in, respectively, $\overline{\mathscr{M}_{\Omega}^{(F)}}$ with the relative weak topology, $\overline{\mathscr{M}_{\Omega}^{(F)} \cap \mathscr{M}_{\Omega}^{B}}$ with the relative strong topology and $\mathscr{M}_{\Omega}^{(F)} \cap \mathscr{M}_{\Omega}^{b}$ with the relative $\tau_{3}$ topology.

Assume $q$ is a positive integer. By Lemma 3.13, for each $\left(\left\{T_{n}\right\}_{n=1}^{\infty}, \gamma\right) \in \mathscr{M}_{\Omega}^{(F)} \times(0,1)$, there exist a positive integer $N_{q}\left(\left\{T_{n}\right\}_{n=1}^{\infty}, \gamma\right)$ and an open neighborhood $U_{q}\left(\left\{T_{n}\right\}_{n=1}^{\infty}, \gamma\right)$ of $\left\{T_{n}^{\gamma}\right\}_{n=1}^{\infty}$ in the space $\mathscr{M}_{\Omega}$ with the weak topology, satisfying the following assertion:

There exists a nonexpansive retraction $Q_{q}\left(\left\{T_{n}\right\}_{n=1}^{\infty}, \gamma\right)$ of $K$ onto $F$ such that, for each $\left\{R_{n}\right\}_{n=1}^{\infty} \in U_{q}\left(\left\{T_{n}\right\}_{n=1}^{\infty}, \gamma\right)$, we have

$$
\left\|R_{N_{q}\left(\left\{T_{n}\right\}_{n=1}^{\infty}, \gamma\right)} \ldots \widetilde{R_{1}} x-Q_{q}\left(\left\{T_{n}\right\}_{n=1}^{\infty}, \gamma\right) x\right\|<\frac{1}{q}
$$

for each $x \in K$.

For each positive integer $q$, set

$$
\begin{gathered}
\mathscr{D}_{q}=\cup_{\left(\left\{T_{n}\right\}_{n=1}^{\infty}, \gamma\right) \in \mathscr{M}_{\Omega}^{(F)} \times(0,1)} U_{q}\left(\left\{T_{n}\right\}_{n=1}^{\infty}, \gamma\right) \cap \overline{\mathscr{M}_{\Omega}^{(F)}}, \\
\mathscr{D}_{q}^{B}=\cup_{\left(\left\{T_{n}\right\}_{n=1}^{\infty}, \gamma\right) \in\left(\mathscr{M}_{\Omega}^{(F)} \cap \mathscr{M}_{\Omega}^{B}\right) \times(0,1)} U_{q}\left(\left\{T_{n}\right\}_{n=1}^{\infty}, \gamma\right) \cap \overline{\mathscr{M}_{\Omega}^{(F)} \cap \mathscr{M}_{\Omega}^{B}} \\
\text { and } \\
\mathscr{D}_{q}^{b}=\cup_{\left(\left\{T_{n}\right\}_{n=1}^{\infty}, \gamma\right) \in\left(\mathscr{M}_{\Omega}^{(F)} \cap \mathscr{M}_{\Omega}^{b}\right) \times(0,1)} U_{q}\left(\left\{T_{n}\right\}_{n=1}^{\infty}, \gamma\right) \cap \mathscr{M}_{\Omega}^{(F)} \cap \mathscr{M}_{\Omega}^{b} .
\end{gathered}
$$

Clearly, the sets $\mathscr{D}_{q}, \mathscr{D}_{q}^{B}$ and $\mathscr{D}_{q}^{b}$ are open (in the relative weak topology) and dense (respectively, in the weak topology, in the relative strong topology and in the relative $\tau_{3}$ topology) sets in, respectively, $\mathscr{M}_{\Omega}^{(F)}, \overline{\mathscr{M}_{\Omega}^{(F)} \cap \mathscr{M}_{\Omega}^{B}}$ and $\mathscr{M}_{\Omega}^{(F)} \cap \mathscr{M}_{\Omega}^{b}$ for each positive integer $q$, because these sets contain, respectively, $A, A^{B}$ and $A^{b}$. Define $\mathscr{F}=\cap_{q=1}^{\infty} \mathscr{D}_{q}, \mathscr{F}^{\prime}=\cap_{q=1}^{\infty} \mathscr{D}_{q}^{B}$ and $\mathscr{F}^{\prime \prime}=\cap_{q=1}^{\infty} \mathscr{D}_{q}^{b}$. Evidently, $\mathscr{F}, \mathscr{F}^{\prime}$ and $\mathscr{F}^{\prime \prime}$ are countable intersections of open (in the relative weak topology) and dense (respectively, in the weak topology, in the relative strong topology and in the relative $\tau_{3}$ topology) sets in, respectively, $\overline{\mathscr{M}_{\Omega}^{(F)}}, \overline{\mathscr{M}_{\Omega}^{(F)} \cap \mathscr{M}_{\Omega}^{B}}$ and $\mathscr{M}_{\Omega}^{(F)} \cap \mathscr{M}_{\Omega}^{b}$.

Assume $\left\{S_{n}\right\}_{n=1}^{\infty} \in \mathscr{F}$ and $\varepsilon>0$. There exist a positive integer $q_{0}$ such that $\frac{2}{q_{0}}<\varepsilon$ and a pair $\left(\left\{T_{n}\right\}_{n=1}^{\infty}, \gamma\right) \in \mathscr{M}_{\Omega}^{(F)} \times(0,1)$ such that $\left\{S_{n}\right\}_{n=1}^{\infty} \in U_{q_{0}}\left(\left\{T_{n}\right\}_{n=1}^{\infty}, \gamma\right)$. Let $\left\{R_{n}\right\}_{n=1}^{\infty} \in$ $U_{q_{0}}\left(\left\{T_{n}\right\}_{n=1}^{\infty}, \gamma\right) \cap \overline{\mathscr{M}_{\Omega}^{(F)}}$. By (4.17), we have

$$
\left\|R_{N_{q_{0}}\left(\left\{T_{n}\right\}_{n=1}^{\infty}, \gamma\right)} \ldots \widetilde{R_{1} x}-Q_{q_{0}}\left(\left\{T_{n}\right\}_{n=1}^{\infty}, \gamma\right) x\right\|<\frac{1}{q_{0}}<2^{-1} \varepsilon
$$


for each $x \in K$. Since $\mathscr{M}^{(F)}$ is closed in $\mathscr{M}$ with the weak topology, by Proposition (1.2), $\left\{\widetilde{R_{n}}\right\}_{n=1}^{\infty} \in \mathscr{M}^{(F)}$ and therefore, by (4.18), for each integer $n \geq N_{q_{0}}\left(\left\{T_{n}\right\}_{n=1}^{\infty}, \gamma\right)$, we have

$$
\left\|\widetilde{R_{n}} \ldots \widetilde{R_{1}} x-Q_{q_{0}}\left(\left\{T_{n}\right\}_{n=1}^{\infty}, \gamma\right) x\right\|<\frac{1}{q_{0}}<2^{-1} \varepsilon
$$

for each $x \in K$. In particular, for each integer $n \geq N_{q_{0}}\left(\left\{T_{n}\right\}_{n=1}^{\infty}, \gamma\right)$, we have

$$
\left\|\widetilde{S_{n}} \ldots \widetilde{S_{1}} x-Q_{q_{0}}\left(\left\{T_{n}\right\}_{n=1}^{\infty}, \gamma\right) x\right\|<\frac{1}{q_{0}}<2^{-1} \varepsilon
$$

for each $x \in K$. Since $\varepsilon$ is an arbitrary positive number, (4.20), the triangle inequality and the completeness of the subspace $K$ of $X$ imply that the sequence of operators $\left\{\widetilde{S}_{n} \ldots \widetilde{S}_{1}\right\}_{n=1}^{\infty}$ converges to an operator $Q: K \rightarrow K$, uniformly on $K$. By switching to the limit in (4.20), we have

$$
\left\|Q x-Q_{q_{0}}\left(\left\{T_{n}\right\}_{n=1}^{\infty}, \gamma\right) x\right\|<2^{-1} \varepsilon
$$

for each $x \in K$. Since $\varepsilon$ is an arbitrary positive number, by (4.21) and the closedness of $F$, we see that the image of $Q$ lies in $F$.

By (4.19), (4.21) and the triangle inequality, we obtain that for each integer $n \geq N_{q_{0}}\left(\left\{T_{n}\right\}_{n=1}^{\infty}, \gamma\right)$,

$$
\left\|\widetilde{R_{n}} \ldots \widetilde{R_{1}} x-Q x\right\| \leq\left\|\widetilde{R_{n}} \ldots \widetilde{R_{1}} x-Q_{q_{0}}\left(\left\{T_{n}\right\}_{n=1}^{\infty}, \gamma\right) x\right\|+\left\|Q_{q_{0}}\left(\left\{T_{n}\right\}_{n=1}^{\infty}, \gamma\right) x-Q x\right\|<\varepsilon
$$

for each $x \in K$. This completes the proof of Theorem 2.6.

The proof of Theorem 2.7 is analogous to that of Theorem 2.6; instead of Lemma 3.13 we use Lemma 3.14.

Remark 4.1. It seems natural to complement our results with an analogue of Theorem 2.2, where we would consider a corresponding statement regarding the space $\mathscr{\mathscr { M }}_{w \Omega}^{\text {reg }}$ (the closure of $\mathscr{M}_{\Omega}^{\text {reg }}$ in $\mathscr{M}_{\Omega}$ with respect to the weak topology) with open (in the weak topology) and dense (in the relative weak topology) sets, $\overline{\mathscr{M}}_{\Omega}^{\text {reg }} \cap \mathscr{M}_{\Omega}^{B}$ with open (in the weak topology) and dense (in the relative strong topology) sets, and $\overline{\mathscr{M}}_{\Omega}^{\text {reg }} \cap \mathscr{M}_{\Omega}^{b}$, with open (in the weak topology) and dense (in the relative $\tau_{3}$ topology) sets. Unfortunately, so far we have not been able to find neither a proof nor a counterexample. Thus we leave this part as an open question for further study.

\section{REFERENCES}

[1] N. Dunford, J. T. Schwartz, Linear Operators, Part I: General Theory, Interscience, New York, 1958.

[2] J. L. Kelley, General Topology, D. Van Nostrand, New York, 1955.

[3] G. Goebel, S. Reich, Uniform Convexity, Hyperbolic Geometry, and Nonexpansive Mappings, Marcel Dekker, New and Basel, 1984.

[4] E. Kopecká, S. Reich, Nonexpansive retracts in Banach spaces, Banach Center Publications 77 (2007), 161174.

[5] S. Reich, A. J. Zaslavski, Genericity in Nonlinear Analysis, Springer, New York, 2014.

[6] M. Gabour, S. Reich, A. J. Zaslavski, Generic convergence of algorithms for solving stochastic feasibility problems, in Inherently Parallel Algorithms in Feasibility and Optimization and Their Applications, pp. 279295, Elsevier, Amsterdam, 2001.

[7] S. Reich, A. J. Zaslavski, Convergence of generic infinite products of nonexpansive and uniformly continuous operators, Nonlinear Anal. 36 (1999), 1049-1065. 